\title{
Vanna-Volga methods applied to FX derivatives: from theory to market practice
}

\author{
Frédéric Bossens§, Grégory Rayée†, Nikos S. Skantzos $₫$ and Griselda Deelstrał \\ $\S$ Termeulenstraat 86A, Sint-Genesius-Rode B-1640, Belgium, frederic.bossens@hotmail.com \\ †Solvay Brussels School of Economics and Management, Université Libre de Bruxelles, \\ Avenue FD Roosevelt 50, CP 165, Brussels 1050, Belgium \\ ФTervuursevest 21 bus 104, Leuven B-3001, Belgium, nikos.skantzos@gmail.com \\ $\ddagger$ Department of Mathematics, Université Libre de Bruxelles, \\ Boulevard du Triomphe, CP 210, Brussels 1050, Belgium
}

May 4, 2010

\begin{abstract}
We study Vanna-Volga methods which are used to price first generation exotic options in the Foreign Exchange market. They are based on a rescaling of the correction to the Black-Scholes price through the so-called 'probability of survival' and the 'expected first exit time'. Since the methods rely heavily on the appropriate treatment of market data we also provide a summary of the relevant conventions. We offer a justification of the core technique for the case of vanilla options and show how to adapt it to the pricing of exotic options. Our results are compared to a large collection of indicative market prices and to more sophisticated models. Finally we propose a simple calibration method based on one-touch prices that allows the Vanna-Volga results to be in line with our pool of market data.
\end{abstract}

\section{Introduction}

The Foreign Exchange (FX) option's market is the largest and most liquid market of options in the world. Currently, the various traded products range from simple vanilla options to first-generation exotics (touch-like options and vanillas with barriers), second-generation exotics (options with a fixingdate structure or options with no available closed form value) and third-generation exotics (hybrid products between different asset classes). Of all the above the first-generation products receive the lion's share of the traded volume. This makes it imperative for any pricing system to provide a fast and accurate mark-to-market for this family of products. Although using the Black-Scholes model [3, 18] it is possible to derive analytical prices for barrier- and touch -options, this model is unfortunately based on several unrealistic assumptions that render the price inaccurate. In particular, the Black-Scholes model assumes that the foreign/domestic interest rates and the FX-spot volatility remain constant throughout the lifetime of the option. This is clearly wrong as these quantities change continuously, reflecting the traders' view on the future of the market. Today the Black-Scholes theoretical value (BS TV) is used only as a reference quotation, to ensure that the involved counterparties are speaking of the same option. 
More realistic models should assume that the foreign/domestic interest rates and the FX spot volatility follow stochastic processes that are coupled to the one of the spot. The choice of the stochastic process depends, among other factors, on empirical observations. For example, for longdated options the effect of the interest rate volatility can become as significant as that of the FX spot volatility. On the other hand, for short-dated options (typically less than 1 year), assuming constant interest rates does not normally lead to significant mispricing. In this article we will assume constant interest rates throughout.

Stochastic volatility models are unfortunately computationally demanding and in most cases require a delicate calibration procedure in order to find the value of parameters that allow the model reproduce the market dynamics. This has led to alternative 'ad-hoc' pricing techniques that give fast results and are simpler to implement, although they often miss the rigor of their stochastic siblings. One such approach is the 'Vanna-Volga' (VV) method that, in a nutshell, consists in adding an analytically derived correction to the Black-Scholes price of the instrument. To do that, the method uses a small number of market quotes for liquid instruments (typically At-The-Money options, Risk Reversal and Butterfly strategies) and constructs an hedging portfolio which zeros out the Black-Scholes Vega, Vanna and Volga of the option. The choice of this set of Greeks is linked to the fact that they all offer a measure of the option's sensitivity with respect to the volatility, and therefore the constructed hedging portfolio aims to take the 'smile' effect into account.

The Vanna-Volga method seems to have first appeared in the literature in [15] where the recipe of adjusting the Black-Scholes value by the hedging portfolio is applied to double-no-touch options and in [27. where it is applied to the pricing of one-touch options in foreign exchange markets. In [15], the authors point out its advantages but also the various pricing inconsistencies that arise from the non-rigorous nature of the technique. The method was discussed more thoroughly in [5] where it is shown that it can be used as a smile interpolation tool to obtain a value of volatility for a given strike while reproducing exactly the market quoted volatilities. It has been further analyzed in [16] where a number of corrections are suggested to handle the pricing inconsistencies. Finally a more rigorous and theoretical justification is given by [17] where, among other directions, the method is extended to include interest-rate risk.

A crucial ingredient to the Vanna-Volga method, that is often overlooked in the literature, is the correct handling of the market data. In FX markets the precise meaning of the broker quotes depends on the details of the contract. This can often lead to treading on thin ice. For instance, there are at least four different definitions for at-the-money strike (resp., 'spot', 'forward', 'delta neutral', '50 delta call'). Using the wrong definition can lead to significant errors in the construction of the smile surface. Therefore, before we begin to explore the effectiveness of the Vanna-Volga technique we will briefly present some of the relevant FX conventions.

The aim of this paper is twofold, namely (i) to describe the Vanna-Volga method and provide an intuitive justification and (ii) to compare its resulting prices against prices provided by renowned FX market makers, and against more sophisticated stochastic models. We attempt to cover a broad range of market conditions by extending our comparison tests into two different 'smile' conditions, one with a mild skew and one with a very high skew. We also describe two variations of the Vanna-Volga method (used by the market) which tend to give more accurate prices when the spot is close to a barrier. We finally describe a simple adjustment procedure that allows the Vanna-Volga method to provide prices that are in good agreement with the market for a wide range of exotic options.

To begin with, in section 2 we describe the set of exotic instruments that we will use in our comparisons throughout. In section 3 we review the market practice of handling market data. Section 4 lays the general ideas underlying the Vanna-Volga adjustment, and proposes an interpretation of 
the method in the context of Plain Vanilla Options. In sections 5.1 and 5.2 we review two common Vanna-Volga variations used to price exotic options. The main idea behind these variations is to reduce Vanna-Volga correction through an attenuation factor. The first one consists in weighting the Vanna-Volga correction by some function of the survival probability, while the second one is based on the so-called expected first exit time argument. Since the Vanna-Volga technique is by no means a self-consistent model, no-arbitrage constraints must be enforced on top of the method. This problem is addressed in section 5.4. In section 5.5 we investigate the sensitivity of the model with respect to the accuracy of the input market data. Finally, Section 6 is devoted to numerical results. After defining a measure of the model error in section 6.1, section 6.2 investigates how the Dupire local vol model [6] and the Heston stochastic vol model [7] perform in pricing. Section 6.3 suggests a simple adaptation that allows the Vanna-Volga method to produce prices reasonably in line with those given by renowned FX platforms. Conclusions of the study are presented in section 7 .

\section{Description of first-generation exotics}

The family of first-generation exotics can be divided into two main subcategories: (i) The hedging options which have a strike and (ii) the treasury options which have no strike and pay a fixed amount. The validity of both types of options at maturity is conditioned on whether the FX-spot has remained below/above the barrier level(s) according to the contract termsheet during the lifetime of the option.

Barrier options can be further classified as either knock-out options or knock-in ones. A knockout option ceases to exist when the underlying asset price reaches a certain barrier level; a knock-in option comes into existence only when the underlying asset price reaches a barrier level. Following the no-arbitrage principle, a knock-out plus a knock-in option (KI) must equal the value of a plain vanilla.

As an example of the first category, we will consider up-and-out calls (UO, also termed Reverse Knock-Out), and double-knock-out calls (DKO). The latter has two knock-out barriers (one up-and-out barrier above the spot level and one down-and-out barrier below the spot level). The exact BlackScholes price of the UO call can be found in [8, 9, 10, while a semi-closed form for double-barrier options is given in [12] in terms of an infinite series (most terms of which are shown to fall to zero very rapidly).

As an example of the second category, we will select one-touch (OT) options paying at maturity one unit amount of currency if the FX-rate ever reaches a pre-specified level during the option's life, and double-one-touch (DOT) options paying at maturity one unit amount of currency if the FX-rate ever reaches any of two pre-specified barrier levels (bracketing the FX-spot from below and above). The Black-Scholes price of the OT option can be found in [20, 25], while the DOT Black-Scholes price is obtained by means of double-knock-in barriers, namely by going long a double-knock-in call spread and a double knock-in put spread.

Although these four types of options represent only a very small fraction of all existing firstgeneration exotics, most of the rest can be obtained by combining the above. This allows us to argue that the results of this study are actually relevant to most of the existing first-generation exotics.

\section{Handling Market Data}

The most famous defect of the Black-Scholes model is the (wrong) assumption that the volatility is constant throughout the lifetime of the option. However, Black-Scholes remains a widespread model 
due to its simplicity and tractability. To adapt it to market reality, if one uses the Black-Scholes formula 1

$$
\begin{aligned}
& \operatorname{Call}(\sigma)=\mathrm{DF}_{d}(t, T)\left[F N\left(d_{1}\right)-K N\left(d_{2}\right)\right] \\
& \operatorname{Put}(\sigma)=-\mathrm{DF}_{d}(t, T)\left[F N\left(-d_{1}\right)-K N\left(-d_{2}\right)\right]
\end{aligned}
$$

in an inverse fashion, giving as input the option's price and receiving as output the volatility, one obtains the so-called 'implied volatility'. Plotting the implied volatility as a function of the strike results typically in a shape that is commonly termed 'smile' (the term 'smile' has been kept for historical reasons, although the shape can be a simple line instead of a smile-looking parabola). The reasons behind the smile effect are mainly that the dynamics of the spot process does not follow a geometric Brownian motion and also that demand for out-of-the-money puts and calls is high (to be used by traders as e.g. protection against market crashes) thereby raising the price, and thus the resulting implied volatility at the edges of the strike domain.

The smile is commonly used as a test-bench for more elaborate stochastic models: any acceptable model for the dynamics of the spot must be able to price vanilla options such that the resulting implied volatilities match the market-quoted ones. The smile depends on the particular currency pair and the maturity of the option. As a consequence, a model that appears suitable for a certain currency pair, may be erroneous for another.

\subsection{Delta conventions}

FX derivative markets use, mainly for historical reasons, the so-called Delta-sticky convention to communicate smile information: the volatilities are quoted in terms of Delta rather than strike value. Practically this means that, if the FX spot rate moves - all other things being equal - the curve of implied volatility vs. Delta will remain unchanged, while the curve of implied volatility vs. strike will shift. Some argue this brings more efficiency in the FX derivatives markets. For a discussion on the appropriateness of the delta-sticky hypothesis we refer the reader to [19]. On the other hand, it makes it necessary to precisely agree upon the meaning of Delta. In general, Delta represents the derivative of the price of an option with respect to the spot. In FX markets, the Delta used to quote volatilities depends on the maturity and the currency pair at hand. An FX spot $S_{t}$ quoted as Ccy1Ccy2 implies that 1 unit of Ccy1 equals $S_{t}$ units of Ccy2. Some currency pairs, mainly those with USD as Ccy2, like EURUSD or GBPUSD, use the Black-Scholes Delta, the derivative of the price with respect to the spot:

$$
\Delta_{\text {call }}=\mathrm{DF}_{f}(t, T) N\left(d_{1}\right) \quad \Delta_{\text {put }}=-\mathrm{DF}_{f}(t, T) N\left(-d_{1}\right)
$$

Setting up the corresponding Delta hedge will make one's position insensitive to small FX spot movements if one is measuring risks in a USD (domestic) risk-neutral world. Other currency pairs (e.g. USDJPY) use the premium included Delta convention:

$$
\tilde{\Delta}_{\text {call }}=\frac{K}{S} \mathrm{DF}_{d}(t, T) N\left(d_{2}\right) \quad \tilde{\Delta}_{\text {put }}=-\frac{K}{S} \mathrm{DF}_{d}(t, T) N\left(-d_{2}\right)
$$

The quantities (2) and (3) are expressed in Ccy1, which is by convention the unit of the quoted Delta. Taking the example of USDJPY, setting up the corresponding Delta hedge (3) will make one's position insensitive to small FX spot movements if one is measuring risks in a USD (foreign) risk-neutral world.

\footnotetext{
${ }^{1}$ for a description of our notation, see $\mathrm{A}$.
} 
Note that (2) and (3) are linked through the option's premium $(1)$, namely $S\left(\Delta_{\text {call }}-\tilde{\Delta}_{\text {call }}\right)=$ Call and similarly for the put (see B for more details).

With regards to the dependency on maturity, the so-called G11 currency pairs use a spot Delta convention (2), (3) for short maturities (typically up to 1 year) while for longer maturities where the interest rate risk becomes significant, the forward Delta (or driftless Delta) is used, as the derivative of the undiscounted premium with respect to forward:

$$
\begin{array}{ll}
\Delta_{\text {call }}^{F}=N\left(d_{1}\right) & \Delta_{\text {put }}^{F}=-N\left(-d_{1}\right) \\
\tilde{\Delta}_{\text {call }}^{F}=\frac{K}{S} \frac{\mathrm{DF}_{d}(t, T)}{\mathrm{DF}_{f}(t, T)} N\left(d_{2}\right) & \tilde{\Delta}_{\text {put }}^{F}=-\frac{K}{S} \frac{\mathrm{DF}_{d}(t, T)}{\mathrm{DF}_{f}(t, T)} N\left(-d_{2}\right)
\end{array}
$$

where, as before, by tilde we denoted the premium-included convention. The Deltas in the first row represent the nominals of the forward contracts to be settled if one is to forward hedge the Delta risk in a domestic currency while those of the second row consider a foreign risk neutral world. Other currency pairs (typically those where interest-rate risks are substantial, even for short maturities) use the forward Delta convention for all maturity pillars.

\subsection{At-The-Money Conventions}

As in the case of the Delta, the at-the-money (ATM) volatilities quoted by brokers can have various interpretations depending on currency pairs. The ATM volatility is the value from the smile curve where the strike is such that the Delta of the call equals, in absolute value, that of the put (this strike is termed ATM 'straddle' or ATM 'delta neutral' ). Solving this equality yields two possible solutions, depending on whether the currency pair uses the Black-Scholes Delta or the premium included Delta convention. The 2 solutions respectively are:

$$
K_{\mathrm{ATM}}=F \exp \left[\frac{1}{2} \sigma_{\mathrm{ATM}}^{2} \tau\right] \quad \tilde{K}_{\mathrm{ATM}}=F \exp \left[-\frac{1}{2} \sigma_{\mathrm{ATM}}^{2} \tau\right]
$$

Note that these expressions are valid for both spot and forward Delta conventions.

\subsection{Smile-related quotes and the broker's Strangle}

Let us assume that a smile surface is available as a function of the strike $\sigma(K)$. In liquid FX markets some of the most traded strategies include

$$
\begin{aligned}
\operatorname{Strangle}\left(K_{c}, K_{p}\right) & =\operatorname{Call}\left(K_{c}, \sigma\left(K_{c}\right)\right)+\operatorname{Put}\left(K_{p}, \sigma\left(K_{p}\right)\right) \\
\operatorname{Straddle}(K) & =\operatorname{Call}\left(K, \sigma_{\mathrm{ATM}}\right)+\operatorname{Put}\left(K, \sigma_{\mathrm{ATM}}\right) \\
\operatorname{Butterfly}\left(K_{p}, K, K_{c}\right) & =\frac{1}{2}\left[\operatorname{Strangle}\left(K_{c}, K_{p}\right)-\operatorname{Straddle}(K)\right]
\end{aligned}
$$

Brokers normally quote volatilities instead of the direct prices of these instruments. These are expressed as functions of $\Delta$, for instance a volatility at $25 \Delta$-call or put refers to the volatility at the strikes $K_{c}, K_{p}$ that satisfy $\Delta_{\text {call }}\left(K_{c}, \sigma\left(K_{c}\right)\right)=0.25$ and $\Delta_{\text {put }}\left(K_{p}, \sigma\left(K_{p}\right)\right)=-0.25$ respectively (with the appropriate Delta conventions, see section 3.1. Typical quotes for the vols are

- at-the-money (ATM) volatility: $\sigma_{\mathrm{ATM}}$

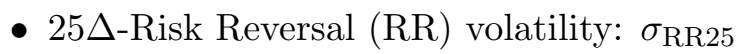


- 1-vol-25 $\Delta$-Butterfly (BF) volatility: $\sigma_{\mathrm{BF} 25(1 \mathrm{vol})}$

- 2-vol-25 $\Delta$-Butterfly $(\mathrm{BF})$ volatility: $\sigma_{\mathrm{BF} 25(2 \mathrm{vol})}$

By market convention, the $\mathrm{RR}$ vol is interpreted as the difference between the call and put implied volatilities respectively:

$$
\sigma_{\mathrm{RR} 25}=\sigma_{25 \Delta C}-\sigma_{25 \Delta P}
$$

where $\sigma_{25 \Delta C}=\sigma\left(K_{c}\right)$ and $\sigma_{25 \Delta P}=\sigma\left(K_{p}\right)$.

The 2-vol-25 $\Delta$-Butterfly can be interpreted through

$$
\sigma_{\mathrm{BF} 25(2 \mathrm{vol})}=\frac{\sigma_{25 \Delta C}+\sigma_{25 \Delta P}}{2}-\sigma_{\mathrm{ATM}}
$$

Associated to the $\sigma_{\mathrm{BF} 25(2 \mathrm{vol})}$ is the 2-vol-25 $\Delta$-strangle vol defined through $\sigma_{\mathrm{STG} 25(2 \mathrm{vol})}=\sigma_{\mathrm{BF} 25(2 \mathrm{vol})}+$ $\sigma_{\mathrm{ATM}}$.

The 2-vol-25 $\Delta$-Butterfly value $\sigma_{\mathrm{BF} 25(2 \mathrm{vol})}$ is in general not directly observable in FX markets. Instead, brokers usually communicate the $\sigma_{\mathrm{BF} 25(1 \mathrm{vol})}$, using a broker's strangle or 1 vol strangle convention. The exact interpretation of $\sigma_{\mathrm{BF} 25(1 \mathrm{vol})}$ can be explained in a few steps:

- Define $\sigma_{\mathrm{STG} 25(1 \mathrm{vol})}=\sigma_{\mathrm{ATM}}+\sigma_{\mathrm{BF} 25(1 \mathrm{vol})}$.

- Solve equations (2), (3) to obtain $K_{25 C}^{*}$ and $K_{25 P}^{*}$, the strikes where the Delta of a call is exactly 0.25 , and the Delta of a put is exactly -0.25 respectively, using the single volatility value $\sigma_{\mathrm{STG} 25(1 \mathrm{vol})}$.

- Provided that the smile curve $\sigma(K)$ is correctly calibrated to the market, then the quoted value $\sigma_{\mathrm{BF} 25(1 \mathrm{vol})}$ is such that the following equality holds:

$$
\operatorname{Call}\left(K_{25 C}^{*}, \sigma_{\mathrm{STG} 25(1 \mathrm{vol})}\right)+\operatorname{Put}\left(K_{25 P}^{*}, \sigma_{\mathrm{STG} 25(1 \mathrm{vol})}\right)=\operatorname{Call}\left(K_{25 C}^{*}, \sigma\left(K_{25 C}^{*}\right)\right)+\operatorname{Put}\left(K_{25 P}^{*}, \sigma\left(K_{25 P}^{*}\right)\right)
$$

The difference between $\sigma_{\mathrm{BF} 25(1 \mathrm{vol})}$ and $\sigma_{\mathrm{BF} 25(2 \mathrm{vol})}$ can be at times confusing. Often for convenience one sets $\sigma_{\mathrm{BF} 25(2 \mathrm{vol})}=\sigma_{\mathrm{BF} 25(1 \mathrm{vol})}$ as this greatly simplifies the procedure to build up a smile curve. However it leads to errors when applied to a steeply skewed market. Figure1 provides a graphical interpretation of the quantities $\sigma_{\mathrm{STG} 25(1 \mathrm{vol})}, \sigma_{\mathrm{STG} 25(2 \mathrm{vol})}, \sigma_{\mathrm{BF} 25(1 \mathrm{vol})}$ and $\sigma_{\mathrm{BF} 25(2 \mathrm{vol})}$ in 2 very different market conditions; the lower panel corresponds to the USDCHF-1Y smile, characterized by a relatively mild skew, the upper panel corresponding to the extremely skewed smile of USDJPY-1Y. As a rule of thumb one sets $\sigma_{\mathrm{BF} 25(2 \mathrm{vol})}=\sigma_{\mathrm{BF} 25(1 \mathrm{vol})}$ when $\sigma_{\mathrm{RR} 25}$ is small in absolute value (typically $\left.<1 \%\right)$. When this empirical condition is not met, $\sigma_{\mathrm{BF} 25(1 \mathrm{vol})}$ and $\sigma_{\mathrm{BF} 25(2 \mathrm{vol})}$ represent actually two different quantities, and substituting one for the other in the context of a smile construction algorithm would yield substantial errors.

Table 1 gives more details about the numerical values used to produce the 2 smiles of Figure 1.

Various differences are observed between the 2 smiles. In the USDCHF case, the values $\sigma_{\mathrm{BF} 25(2 \mathrm{vol})}$ and $\sigma_{\mathrm{BF} 25(1 \mathrm{vol})}$ are close to each other. Similarly, the strikes used in the 1vol-25 $\Delta$ Strangle are rather close to those attached to the 2vol-25 $\Delta$ Strangle. On the contrary, in the USDJPY case, large dif-

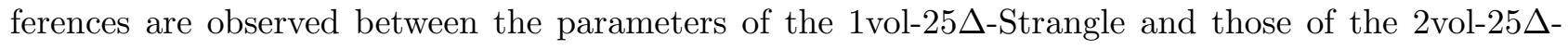
Strangle.

Unfortunately, there is no direct mapping between $\sigma_{\mathrm{BF} 25(1 \mathrm{vol})}$ and $\sigma_{\mathrm{BF} 25(2 \mathrm{vol})}$. This is mainly due to the fact that these two instruments are attached to different points of the implied volatility curve. The relationship between $\sigma_{\mathrm{BF} 25(2 \mathrm{vol})}$ and $\sigma_{\mathrm{BF} 25(1 \mathrm{vol})}$ implicitly depends on the entire smile curve. 

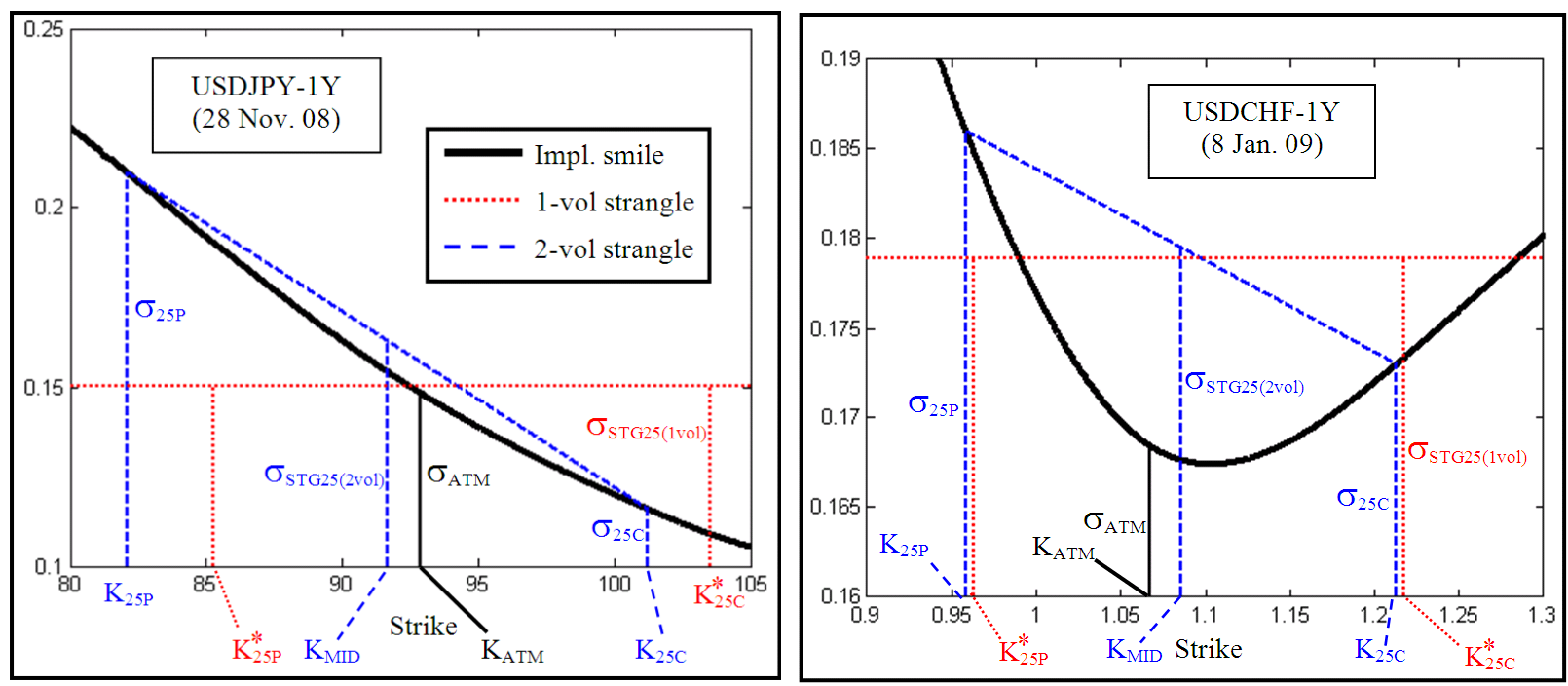

Figure 1: Comparison between $\sigma_{\mathrm{STG} 25(2 \mathrm{vol})}$ and $\sigma_{\mathrm{STG} 25(1 \mathrm{vol})}$, also called 'broker strangle' in two different smile conditions.

\begin{tabular}{|c|c|c|}
\cline { 2 - 3 } \multicolumn{1}{c|}{} & USDCHF & USDJPY \\
\hline date & 8 Jan 09 & 28 Nov 08 \\
\hline FX spot rate & 1.0902 & 95.47 \\
\hline maturity & \multicolumn{2}{|c|}{1 year } \\
\hline$r_{d}$ & $1.3 \%$ & $1.74 \%$ \\
\hline$r_{f}$ & $2.03 \%$ & $3.74 \%$ \\
\hline$\sigma_{A T M}$ & $16.85 \%$ & $14.85 \%$ \\
\hline$\sigma_{\mathrm{RR} 25}$ & $-1.3 \%$ & $-9.4 \%$ \\
\hline$\sigma_{\mathrm{BF} 25(2 \mathrm{vol})}$ & $1.1 \%$ & $1.45 \%$ \\
\hline$\sigma_{\mathrm{BF} 25(1 \mathrm{vol})}$ & $1.04 \%$ & $0.2 \%$ \\
\hline$K_{25 P} / K_{25 C}$ & $0.9586 / 1.2132$ & $82.28 / 101.25$ \\
\hline$K_{25 P}^{*} / K_{25 C}^{*}$ & $0.9630 / 1.2179$ & $85.24 / 103.53$ \\
\hline
\end{tabular}

Table 1: Details of market quotes for the two smile curves of Figure 1.

In practice however, one may be interested in finding the value of $\sigma_{\mathrm{BF} 25(1 \mathrm{vol})}$ from an existing smile curve; this can be achieved using an iterative procedure:

pseudo-algorithm 1

1. Select an initial guess for $\sigma_{\mathrm{BF} 25(1 \mathrm{vol})}$

2. compute the corresponding strikes $K_{25 P}^{*}$ and $K_{25 C}^{*}$

3. assess the validity of equality (11): compare the value of the Strangle (i) valued with a unique vol $\sigma_{\mathrm{BF} 25(1 \mathrm{vol})}\left(\right.$ ii) valued with 2 implied vol corresponding to $K_{25 P}^{*}$, respectively $K_{25 C}^{*}$ 
4. If the difference between the two values exceeds some tolerance level, adapt the value $\sigma_{\mathrm{BF} 25(1 \mathrm{vol})}$ and go back to 2 .

In case one is given a value of $\sigma_{\mathrm{BF} 25(1 \mathrm{vol})}$ from the market, and wants to use it to build an implied smile curve, one may proceed the following way:

pseudo-algorithm 2

1. Select an initial guess for $\sigma_{\mathrm{BF} 25(2 \mathrm{vol})}$

2. Construct an implied smile curve using $\sigma_{\mathrm{BF} 25(2 \mathrm{vol})}$ and market value of $\sigma_{\mathrm{RR} 25}$

3. Compute the value of $\sigma_{\mathrm{BF} 25(1 \mathrm{vol})}$ (for instance following guidelines of pseudo-algorithm 1)

4. Compare $\sigma_{\mathrm{BF} 25(1 \mathrm{vol})}$ you obtained in 3 to the market-given one.

5. If the difference between the two values exceeds some tolerance, adapt the value $\sigma_{\mathrm{BF} 25(2 \mathrm{vol})}$ and go back to 2 .

To close this section on the broker's Strangle issue, let us clarify another enigmatic concept of FX markets often used by practitioners, the so-called Vega-weighted Strangle quote. This is in fact an approximation for the value of $\sigma_{\mathrm{STG} 25(1 \mathrm{vol})}$. To show this, we start from equality (11). First we assume $K_{25 P}^{*}=K_{25 P}$ and $K_{25 C}^{*}=K_{25 C}$. Next, we develop both sides in a first order Taylor expansion in $\sigma$ around $\sigma_{A T M}$. After canceling repeating terms on the left and right-hand side, we are left with:

$$
\begin{aligned}
& \left(\sigma_{\mathrm{STG} 25(1 \mathrm{vol})}-\sigma_{\mathrm{ATM}}\right) \cdot\left(\mathcal{V}\left(K_{25 P}, \sigma_{\mathrm{ATM}}\right)+\mathcal{V}\left(K_{25 C}, \sigma_{\mathrm{ATM}}\right)\right) \\
& \approx\left(\sigma_{25 \Delta P}-\sigma_{\mathrm{ATM}}\right) \cdot \mathcal{V}\left(K_{25 P}, \sigma_{\mathrm{ATM}}\right)+\left(\sigma_{25 \Delta C}-\sigma_{\mathrm{ATM}}\right) \cdot \mathcal{V}\left(K_{25 C}, \sigma_{\mathrm{ATM}}\right)
\end{aligned}
$$

where $\mathcal{V}(K, \sigma)$ represents the Vega of the option, namely the sensitivity of the option price $P$ with respect to a change of the implied volatility: $\mathcal{V}=\frac{\partial P}{\partial \sigma}$. Solving this for $\sigma_{\mathrm{STG} 25(1 \mathrm{vol})}$ yields:

$$
\sigma_{\mathrm{STG} 25(1 \mathrm{vol})} \approx \frac{\sigma_{25 \Delta \mathrm{P}} \cdot \mathcal{V}\left(K_{25 P}, \sigma_{\mathrm{ATM}}\right)+\sigma_{25 \Delta \mathrm{C}} \cdot \mathcal{V}\left(K_{25 C}, \sigma_{\mathrm{ATM}}\right)}{\mathcal{V}\left(K_{25 P}, \sigma_{\mathrm{ATM}}\right)+\mathcal{V}\left(K_{25 C}, \sigma_{\mathrm{ATM}}\right)}
$$

which corresponds to the average (weighted by Vega) of the call and put implied volatilities.

Note that according to Castagna et al. [5] practitioners also use the term Vega-weighted butterfly for a structure where a strangle is bought and an amount of ATM straddle is sold such that the overall vega of the structure is zero.

\section{The Vanna-Volga Method}

The Vanna-Volga method consists in adjusting the Black-Scholes TV by the cost of a portfolio which hedges three main risks associated to the volatility of the option, the Vega, the Vanna and the Volga. The Vanna is the sensitivity of the Vega with respect to a change in the spot FX rate: Vanna $=\frac{\partial \mathcal{V}}{\partial S}$. Similarly, the Volga is the sensitivity of the Vega with respect to a change of the implied volatility $\sigma$ : Volga $=\frac{\partial \mathcal{V}}{\partial \sigma}$. The hedging portfolio will be composed of the following three strategies:

$$
\begin{aligned}
\mathrm{ATM} & =\frac{1}{2} \operatorname{Straddle}\left(K_{\mathrm{ATM}}\right) \\
\mathrm{RR} & =\operatorname{Call}\left(K_{c}, \sigma\left(K_{c}\right)\right)-\operatorname{Put}\left(K_{p}, \sigma\left(K_{p}\right)\right) \\
\mathrm{BF} & =\frac{1}{2} \operatorname{Strangle}\left(K_{c}, K_{p}\right)-\frac{1}{2} \operatorname{Straddle}\left(K_{\mathrm{ATM}}\right)
\end{aligned}
$$


where $K_{\mathrm{ATM}}$ represents the ATM strike, $K_{c / p}$ the 25 -Delta call/put strikes obtained by solving the equations $\Delta_{\text {call }}\left(K_{c}, \sigma_{\mathrm{ATM}}\right)=\frac{1}{4}$ and $\Delta_{\text {put }}\left(K_{p}, \sigma_{\mathrm{ATM}}\right)=-\frac{1}{4}$ and $\sigma\left(K_{c / p}\right)$ the corresponding volatilities evaluated from the smile surface.

\subsection{The general framework}

In this section we present the Vanna-Volga methodology.

The simplest formulation [25] suggests that the Vanna-Volga price $X^{\mathrm{VV}}$ of an exotic instrument $X$ is given by

$$
X^{\mathrm{VV}}=X^{\mathrm{BS}}+\underbrace{\frac{\operatorname{Vanna}(X)}{\operatorname{Vanna}(\mathrm{RR})}}_{w_{\mathrm{RR}}} \mathrm{RR}_{\text {cost }}+\underbrace{\operatorname{Volga}(X)}_{w_{\mathrm{BF}}} \mathrm{BF}_{\text {cost }}
$$

where by $X^{\mathrm{BS}}$ we denoted the Black-Scholes price of the exotic and the Greeks are calculated with ATM volatility. Also, for any instrument $I$ we define its 'smile cost' as the difference between its price computed with/without including the smile effect: $I_{\text {cost }}=I_{\mathrm{mkt}}-I_{\mathrm{BS}}$, and in particular

$$
\begin{aligned}
\mathrm{RR}_{\text {cost }} & =\left[\operatorname{Call}\left(K_{c}, \sigma\left(K_{c}\right)\right)-\operatorname{Put}\left(K_{p}, \sigma\left(K_{p}\right)\right)\right]-\left[\operatorname{Call}\left(K_{c}, \sigma_{\mathrm{ATM}}\right)-\operatorname{Put}\left(K_{p}, \sigma_{\mathrm{ATM}}\right)\right] \\
\mathrm{BF}_{\text {cost }} & =\frac{1}{2}\left[\operatorname{Call}\left(K_{c}, \sigma\left(K_{c}\right)\right)+\operatorname{Put}\left(K_{p}, \sigma\left(K_{p}\right)\right)\right]-\frac{1}{2}\left[\operatorname{Call}\left(K_{c}, \sigma_{\mathrm{ATM}}\right)+\operatorname{Put}\left(K_{p}, \sigma_{\mathrm{ATM}}\right)\right]
\end{aligned}
$$

The rationale behind (15) is that one can extract the smile cost of an exotic option by measuring the smile cost of a portfolio designed to hedge its Vanna and Volga risks. The reason why one chooses the strategies BF and RR to do this is because they are liquid FX instruments and they carry respectively mainly Volga and Vanna risks. The weighting factors $w_{R R}$ and $w_{B F}$ in (15) represent respectively the amount of RR needed to replicate the option's Vanna, and the amount of BF needed to replicate the option's Volga. The above approach ignores the small (but non-zero) fraction of Volga carried by the RR and the small fraction of Vanna carried by the BF. It further neglects the cost of hedging the Vega risk. This has led to a more general formulation of the Vanna-Volga method [5] in which one considers that within the BS assumptions the exotic option's Vega, Vanna and Volga can be replicated by the weighted sum of three instruments:

$$
\vec{x}=\mathbb{A} \vec{w}
$$

with

$$
\mathbb{A}=\left(\begin{array}{ccc}
\mathrm{ATM}_{\text {vega }} & \mathrm{RR}_{\text {vega }} & \mathrm{BF}_{\text {vega }} \\
\mathrm{ATM}_{\text {vanna }} & \mathrm{RR}_{\text {vanna }} & \mathrm{BF}_{\text {vanna }} \\
\mathrm{ATM}_{\text {volga }} & \mathrm{RR}_{\text {volga }} & \mathrm{BF}_{\text {volga }}
\end{array}\right) \quad \vec{w}=\left(\begin{array}{c}
w_{\mathrm{ATM}} \\
w_{\mathrm{RR}} \\
w_{\mathrm{BF}}
\end{array}\right) \quad \vec{x}=\left(\begin{array}{c}
X_{\text {vega }} \\
X_{\text {vanna }} \\
X_{\text {volga }}
\end{array}\right)
$$

the weightings $\vec{w}$ are to be found by solving the systems of equations (17).

Given this replication, the Vanna-Volga method adjusts the BS price of an exotic option by the smile cost of the above weighted sum (note that the ATM smile cost is zero by construction):

$$
\begin{aligned}
X^{\mathrm{VV}} & =X^{\mathrm{BS}}+w_{\mathrm{RR}}\left(\mathrm{RR}^{\mathrm{mkt}}-\mathrm{RR}^{\mathrm{BS}}\right)+w_{\mathrm{BF}}\left(\mathrm{BF}^{\mathrm{mkt}}-\mathrm{BF}^{\mathrm{BS}}\right) \\
& =X^{\mathrm{BS}}+\vec{x}^{T}\left(\mathbb{A}^{T}\right)^{-1} \vec{I}=X^{\mathrm{BS}}+X_{\text {vega }} \Omega_{\text {vega }}+X_{\text {vanna }} \Omega_{\mathrm{vanna}}+X_{\mathrm{volga}} \Omega_{\mathrm{volga}}
\end{aligned}
$$


where

$$
\vec{I}=\left(\begin{array}{c}
0 \\
\mathrm{RR}^{\mathrm{mkt}}-\mathrm{RR}^{\mathrm{BS}} \\
\mathrm{BF}^{\mathrm{mkt}}-\mathrm{BF}^{\mathrm{BS}}
\end{array}\right) \quad\left(\begin{array}{c}
\Omega_{\mathrm{vega}} \\
\Omega_{\mathrm{vanna}} \\
\Omega_{\mathrm{volga}}
\end{array}\right)=\left(\mathbb{A}^{T}\right)^{-1} \vec{I}
$$

and where the quantities $\Omega_{i}$ can be interpreted as the market prices attached to a unit amount of Vega, Vanna and Volga, respectively. For vanillas this gives a very good approximation of the market price. For exotics, however, e.g. no-touch options close to a barrier, the resulting correction typically turns out to be too large. Following market practice we thus modify 19 to

$$
X^{\mathrm{VV}}=X^{\mathrm{BS}}+p_{\mathrm{vanna}} X_{\mathrm{vanna}} \Omega_{\mathrm{vanna}}+p_{\mathrm{volga}} X_{\mathrm{volga}} \Omega_{\mathrm{volga}}
$$

where we have dropped the Vega contribution which turns out to be several orders of magnitude smaller than the Vanna and Volga terms in all practical situations, and where $p_{\text {vanna }}$ and $p_{\text {volga }}$ represent attenuation factors which are functions of either the 'survival probability' or the expected 'first-exit time'. We will return to these concepts in section 5 .

\subsection{Vanna-Volga as a smile-interpolation method}

In [5], Castagna and Mercurio show how Vanna-Volga can be used as a smile interpolation method. They give an elegant closed-form solution (unique) of system (17), when $X$ is a European call or put with strike $K$.

In their paper they adjust the Black Scholes price by using a replicating portfolio composed of a weighted sum of three vanillas (calls or puts) struck respectively at $K_{1}, K_{2}$ and $K_{3}$, where $K_{1}<K_{2}<$ $K_{3}$. They show that the weights $w_{i}$ associated to the vanillas struck at $K_{i}$ such that the resulting portfolio hedges the Vega, Vanna and Volga risks of the vanilla of strike $K$ are unique and given by:

$$
\begin{aligned}
w_{1}(K) & =\frac{\operatorname{Vega}(K)}{\operatorname{Vega}\left(K_{1}\right)} \frac{\ln \frac{K_{2}}{K} \ln \frac{K_{3}}{K}}{\ln \frac{K_{2}}{K_{1}} \ln \frac{K_{3}}{K_{1}}} \\
w_{2}(K) & =\frac{\operatorname{Vega}(K)}{\operatorname{Vega}\left(K_{2}\right)} \frac{\ln \frac{K}{K_{1}} \ln \frac{K_{3}}{K}}{\ln \frac{K_{2}}{K_{1}} \ln \frac{K_{3}}{K_{2}}} \\
w_{3}(K) & =\frac{\operatorname{Vega}(K)}{\operatorname{Vega}\left(K_{3}\right)} \frac{\ln \frac{K}{K_{1}} \ln \frac{K}{K_{2}}}{\ln \frac{K_{3}}{K_{1}} \ln \frac{K_{3}}{K_{2}}}
\end{aligned}
$$

The fact that this solution provides an exact interpolation method is easily verified by noticing that $w_{i}\left(K_{i}\right)=1$ and $w_{i}\left(K_{j}\right)=0, i \neq j$.

This solution still holds in the case of a replicating portfolio composed of ATM, RR and BF instruments as described in section 4 by equations (14). Setting $K_{1}=K_{p}, K_{2}=K_{\text {ATM }}$ and $K_{3}=K_{c}$, a simple coordinate transform yields:

$$
\begin{aligned}
w_{\mathrm{ATM}}(K) & =w_{1}(K)+w_{2}(K)+w_{3}(K) \\
w_{\mathrm{RR}}(K) & =\frac{1}{2}\left(w_{3}(K)-w_{1}(K)\right) \\
w_{\mathrm{BF}}(K) & =w_{1}(K)+w_{3}(K)
\end{aligned}
$$

where the weights $w_{\mathrm{ATM}}, w_{\mathrm{RR}}$ and $w_{\mathrm{BF}}$ are defined by (17)- 18 . 
We now turn back to the elementary Vanna-Volga recipe (15). Unlike the previously exposed exact solution, it does not reproduce the market price of $\mathrm{RR}$ and $\mathrm{BF}$, a fortiori is it not an interpolation method for plain vanillas. However, this approximation possesses the merit of allowing a qualitative interpretation of the $\mathrm{RR}$ and $\mathrm{BF}$ correction terms in (15).

As we will demonstrate, those two terms directly relate to the slope and convexity of the smile curve. To start with, we introduce a new smile parametrization variable:

$$
Y=\ln \frac{K}{F \cdot \exp \left(\frac{1}{2} \sigma_{\mathrm{ATM}}^{2} \tau\right)}=\ln \frac{K}{K_{\mathrm{ATM}}}
$$

Note that the Vega of a Plain Vanilla Option is a symmetric function of $Y$ :

$$
\operatorname{Vega}(Y)=\operatorname{Vega}(-Y)=S e^{-r_{f} \tau} \sqrt{\tau} n\left(\frac{Y}{\sigma \sqrt{\tau}}\right)
$$

where $n(\cdot)$ denotes the Normal density function.

Let us further assume that the smile curve is a quadratic function of $Y$ :

$$
\sigma(Y)=\sigma_{\mathrm{ATM}}+b Y+c Y^{2}
$$

In this way we allow the smile to have a skew (linear term) and a curvature (quadratic term), while keeping an analytically tractable expression. We now express Vanna and Volga of Plain Vanilla Options as functions of $Y$ :

$$
\begin{aligned}
\operatorname{Vanna}(Y) & =\operatorname{Vega}(Y) \cdot \frac{Y+\sigma^{2} \tau}{S \sigma^{2} \tau} \\
\operatorname{Volga}(Y) & =\operatorname{Vega}(Y) \cdot \frac{Y^{2}+\sigma^{2} \tau Y}{\sigma^{3} \tau}
\end{aligned}
$$

Working with the plain Black-Scholes Delta (2) and the delta-neutral ATM definition and defining $Y_{i}=\ln \frac{K_{i}}{K_{\mathrm{ATM}}}$ we have that $Y_{A T M}$ and $Y_{25 P}$ and $Y_{25 C}$ corresponding respectively to At-The-Money, 25-Delta Put, and 25-Delta Call solve

$$
\begin{aligned}
& Y_{A T M}=0 \\
& \operatorname{DF}_{f}(t, T) N\left(\frac{Y_{25 P}-\frac{1}{2}\left(\sigma_{25 P}^{2}-\sigma_{A T M}^{2}\right) \tau}{\sigma_{25 P} \sqrt{\tau}}\right)=\frac{1}{4} \\
& \operatorname{DF}_{f}(t, T) N\left(\frac{-Y_{25 C}+\frac{1}{2}\left(\sigma_{25 C}^{2}-\sigma_{A T M}^{2}\right) \tau}{\sigma_{25 C} \sqrt{\tau}}\right)=\frac{1}{4}
\end{aligned}
$$

Under the assumption that $\sigma_{25 \Delta C} \approx \sigma_{25 \Delta P} \approx \sigma_{A T M}$ we find $Y_{25 C} \approx-Y_{25 P}$. In this case using equations (25) and (14), the Vanna of the RR and the Volga of the BF can be expressed as :

$$
\begin{aligned}
& \operatorname{Vanna}(R R)=\operatorname{Vanna}\left(Y_{25 C}\right)-\operatorname{Vanna}\left(Y_{25 P}\right)=2 \frac{\operatorname{Vega}\left(Y_{25 C}\right) Y_{25 C}}{S \cdot \sigma_{\text {ATM }}^{2} \cdot \tau} \\
& \operatorname{Volga}(B F)=\frac{\operatorname{Volga}\left(Y_{25 C}\right)+\operatorname{Volga}\left(Y_{25 P}\right)}{2}-\operatorname{Volga}(0)=\frac{\operatorname{Vega}\left(Y_{25 C}\right) Y_{25 C}^{2}}{\sigma_{\text {ATM }}^{3}}
\end{aligned}
$$


To calculate $\mathrm{RR}_{\text {cost }}$ and $\mathrm{BF}_{\text {cost }}$ (the difference between the price calculated with smile, and that calculated with a constant volatility $\sigma_{\mathrm{ATM}}$ ), we introduce the following convenient approximation:

$$
\operatorname{Call}(\sigma(Y))-\operatorname{Call}\left(\sigma_{\mathrm{ATM}}\right) \approx \operatorname{Vega}(Y) \cdot\left(\sigma(Y)-\sigma_{\mathrm{ATM}}\right)
$$

using the above, it is straightforward to show that:

$$
\begin{aligned}
& R R_{\text {cost }} \approx 2 b \operatorname{Vega}\left(Y_{25 C}\right) Y_{25 C} \\
& B F_{\text {cost }} \approx c \operatorname{Vega}\left(Y_{25 C}\right) Y_{25 C}^{2}
\end{aligned}
$$

Substituting expressions (25), (26) and (28) in the simple VV recipe (15) yields the following remarkably simple result:

$$
\begin{aligned}
X^{\mathrm{VV}}(Y) & =X^{\mathrm{BS}}(Y)+\frac{\operatorname{Vanna}(Y)}{\operatorname{Vanna}(\mathrm{RR})} \mathrm{RR}_{\mathrm{cost}}+\frac{\operatorname{Volga}(Y)}{\operatorname{Volga}(\mathrm{BF})} \mathrm{BF}_{\text {cost }} \\
& \approx X^{\mathrm{BS}}+\operatorname{Vega}(\mathrm{Y}) b Y+\operatorname{Vega}(\mathrm{Y}) c Y^{2}+\operatorname{Vega}(\mathrm{Y}) \sigma_{\mathrm{ATM}}^{2} \tau \cdot(b+c Y) \\
& =X^{\mathrm{BS}}(Y)+\frac{\partial X^{\mathrm{BS}}}{\partial \sigma}(Y) \cdot\left(\sigma(Y)-\sigma_{\mathrm{ATM}}\right)+\operatorname{Vega}(\mathrm{Y}) \sigma_{\mathrm{ATM}}^{2} \tau \cdot(b+c Y)
\end{aligned}
$$

Despite the presence of a residual term, which vanishes as $\tau \rightarrow 0$ or $\sigma_{\text {ATM }} \rightarrow 0$, the above expression shows that the Vanna-Volga price (15) of a vanilla option can be written as a first-order Taylor expansion of the BS price around $\sigma_{\mathrm{ATM}}$. Furthermore, as $\frac{\operatorname{Vanna}(Y)}{\operatorname{Vanna}(\mathrm{RR})} \mathrm{RR}_{\text {cost }} \approx \operatorname{Vega}(\mathrm{Y}) b Y$ and $\frac{\operatorname{Volga}(Y)}{\operatorname{Volga}(\mathrm{BF})} \mathrm{BF}_{\text {cost }} \approx \operatorname{Vega}(\mathrm{Y}) c Y^{2}$, the RR term (coupled to Vanna) accounts for the impact of the linear component of the smile on the price, while the BF (coupled to Volga) accounts for the impact of the quadratic component of the smile on the price.

\section{Market-adapted variations of Vanna-Volga}

In this section we describe two empirical ways of adjusting the weights $\left(p_{\text {vanna }}, p_{\text {volga }}\right)$ in 21 . We will focus our attention on knock-out options, although the Vanna-Volga approach can be readily generalized to options containing knock-in barriers, as those can always be decomposed into two knock-out (or vanilla) ones (through the no-arbitrage relation knock-in = vanilla - knock-out).

To justify the need for the correction factors to (21) we argue as follows: As the knock-out barrier level $B$ of an option is gradually moved toward the spot level $S_{t}$, the BS price of a KO option must be a monotonically decreasing function, converging to zero exactly at $B=S_{t}$. Since the Vanna-Volga method is a simple rule-of-thumb and not a rigorous model, there is no guarantee that this will be satisfied. We thus have to impose it through the attenuations factors $p_{\text {vanna }}$ and $p_{\text {volga }}$. Note that for barrier values close to the spot, the Vanna and the Volga behave differently: the Vanna becomes large while, on the contrary, the Volga becomes small. Hence we seek attenuation factors of the form:

$$
p_{\mathrm{vanna}}=a \gamma \quad p_{\mathrm{volga}}=b+c \gamma
$$

where $\gamma \in[0,1]$ represents some measure of the barrier(s) vicinity to the spot with the features

$$
\begin{array}{lll}
\gamma=0 & \text { for } & S_{t} \rightarrow B \\
\gamma=1 & \text { for } & \left|S_{t}-B\right| \gg 0
\end{array}
$$


i.e. the limiting cases refer to the regions where the spot is close versus away from the barrier level. Before moving to more specific definitions of $\gamma$, let us introduce some restrictions on $p_{\text {vanna }}$ and $p_{\text {volga }}$ :

$$
\lim _{\gamma \rightarrow 1} p_{\text {vanna }}=1 \quad \lim _{\gamma \rightarrow 1} p_{\text {volga }}=1
$$

The above conditions ensure that when the barrier is far from the spot, implying that hitting the barrier becomes very unlikely, the Vanna-Volga algorithm boils down to its simplest form (15) which is a good approximation to the price of a vanilla option using the market quoted volatility. We therefore amend the expressions 30 into continuous, piecewise linear functions:

$$
p_{\text {vanna }}=\left\{\begin{array}{ll}
a \gamma & \gamma \leq \gamma^{*} \\
a \gamma^{*} \frac{1-\gamma}{1-\gamma^{*}}+\frac{\gamma-\gamma^{*}}{1-\gamma^{*}} & \gamma>\gamma^{*}
\end{array} \quad p_{\mathrm{volga}}= \begin{cases}b+c \gamma & \gamma \leq \gamma^{*} \\
\left(b+c \gamma^{*}\right) \frac{1-\gamma}{1-\gamma^{*}}+\frac{\gamma-\gamma^{*}}{1-\gamma^{*}} \quad \gamma>\gamma^{*}\end{cases}\right.
$$

where $\gamma^{*}$ is a transition threshold chosen close to 1 . Note that the amendment (34) is justified only in the case of options that degenerate into plain vanilla instruments in the region where the barriers are away from the spot. However, in the case of treasury options that do not have a strike (e.g. OT), there is no smile effect in the region where the barriers are away from the spot as these options pay a fixed amount and their fair value is provided by the BS TV. In this case, no amendment is necessary as both Vanna and Volga go to zero.

We now proceed to specify practical $\gamma$ candidates, namely the survival probability and the expected first exit time (FET). In what follows, the corresponding Vanna-Volga prices will be denoted by $\mathrm{VV}_{\text {surv }}$ and $\mathrm{VV}_{\text {fet }}$ respectively.

\subsection{Survival probability}

The survival probability $p_{\text {surv }} \in[0,1]$ refers to the probability that the spot does not touch one or more barrier levels before the expiry of the option. Here we need to distinguish whether the spot process is simulated through the domestic or the foreign risk-neutral measures:

$$
\begin{aligned}
\text { domestic : } & d S_{t}=S_{t}\left(r_{d}-r_{f}\right) d t+S_{t} \sigma d W_{t} \\
\text { foreign : } & d S_{t}=S_{t}\left(r_{d}-r_{f}+\sigma^{2}\right) d t+S_{t} \sigma d W_{t}
\end{aligned}
$$

where $W_{t}$ is a Wiener process. One notices that the quanto drift adjustment will obviously have an impact in the value of the survival probability. Then, for e.g. a single barrier option we have

$$
\begin{aligned}
\text { domestic : } & p_{\text {surv }}^{d}=\mathrm{E}^{d}\left[1_{S_{t^{\prime}}<B, t<t^{\prime}<T}\right]=\mathrm{NT}^{d}(B) / \mathrm{DF}_{d}(t, T) \\
\text { foreign : } & p_{\text {surv }}^{f}=\mathrm{E}^{f}\left[1_{S_{t^{\prime}}<B, t<t^{\prime}<T}\right]=\mathrm{NT}^{f}(B) / \mathrm{DF}_{f}(t, T)
\end{aligned}
$$

where $\mathrm{NT}^{d / f}(B)$ is the value of a no-touch option in the domestic/foreign measure, $\mathrm{E}^{d / f}$ is the risk neutral expectation in the domestic/foreign market respectively, and $1_{a}$ is the indicator function for the event "a". Similarly, for options with two barriers the survival probability is given through the undiscounted value of a double-no-touch option. Explicit formulas for no-touch and double-no-touch options can be found in [25].

The survival probability clearly satisfies the required features (31), (32). To respect domestic/foreign symmetries we further define $\gamma_{\text {surv }}=\frac{1}{2}\left(p_{\text {surv }}^{d}+p_{\text {surv }}^{f}\right)$. 


\subsection{First exit time}

The first exit time is the minimum between: (i) the time in the future when the spot is expected to exit a barrier zone before maturity, and (ii) maturity, if the spot has not hit any of the barrier levels up to maturity. That is, if we denote the FET by $u\left(S_{t}, t\right)$ then $u\left(S_{t}, t\right)=\min \{\phi, \tau\}$ where $\phi=\inf \left\{\ell \in[0, \infty) \mid S_{t+\ell}>H\right.$ or $\left.S_{t+\ell}<L\right\}$ where $L<S_{t}<H$ define the barrier levels, $S_{t}$ the spot of today and $\tau$ the time to maturity (expressed in years). This quantity also has the desirable feature that it becomes small near a barrier and can therefore be used to rescale the two correction terms in (21).

Let us give some definitions. For a geometric Brownian motion spot process of constant volatility $\sigma$ and drift $\mu$, the cumulative probability of the spot hitting a barrier between $t^{*}$ and $t^{\prime}\left(t<t^{*}<T\right.$, $\left.t^{\prime}>t^{*}\right)$ denoted by $C\left(S, t^{*}, t^{\prime}\right)$ obeys a backward Kolmogorov equation [24] (in fact $C\left(S, t^{*}, t^{\prime}\right)$ can be thought of as the undiscounted price of a DOT option):

$$
\mathcal{F} C=0 \quad \mathcal{F} \equiv \frac{\partial}{\partial t^{*}}+\frac{1}{2} \sigma^{2} S^{2} \frac{\partial^{2}}{\partial S^{2}}+\mu S \frac{\partial}{\partial S}
$$

with boundary conditions $C\left(L, t^{*}, t^{\prime}\right)=C\left(H, t^{*}, t^{\prime}\right)=1$ and $C\left(S, t^{\prime}, t^{\prime}\right)=0$ assuming that there are no window-barrier $2^{2}$. Now suppose that at some time $t^{*}>t$, we are standing at $S$, and no barrier was hit so far, the expected FET (measured from $t$ ) is then by definition:

$$
\overline{u\left(S, t^{*}\right)}=t^{*}-t+\int_{t^{*}}^{T}\left(t^{\prime}-t^{*}\right) \frac{\partial C}{\partial t^{\prime}} d t^{\prime}+\int_{T}^{\infty}\left(T-t^{*}\right) \frac{\partial C}{\partial t^{\prime}} d t^{\prime}
$$

while integration by parts gives

$$
\overline{u\left(S, t^{*}\right)}=t^{*}-t+\int_{t^{*}}^{T}\left(1-C\left(S, t^{*}, t^{\prime}\right)\right) d t^{\prime}
$$

and finally taking derivative with respect to $t^{*}$, and first and second derivatives with respect to $S$ and integrating (39) from $t^{*}$ to $T$ results in:

$$
\frac{\partial \bar{u}}{\partial t^{*}}+\frac{1}{2} \sigma^{2} S^{2} \frac{\partial^{2} \bar{u}}{\partial S^{2}}+\mu S \frac{\partial \bar{u}}{\partial S}=0 \Leftrightarrow \mathcal{F} \bar{u}=0
$$

note that this is slightly different from the expression in [24, where FET is measured from $t^{*}$. Equation (42) is solved backwards in time from $t^{*}=T$ to $t^{*}=t$, starting from the terminal condition $\overline{u(S, T)}=\tau$ and boundary conditions $\overline{u\left(L, t^{*}\right)}=\overline{u\left(H, t^{*}\right)}=t^{*}-t$. In case of a single barrier option we use the same PDE with either $H \gg S_{t}$ or $L \ll S_{t}$.

As for the case of the survival probability we solve the PDE (42) in both the domestic and foreign risk-neutral cases which implies that we set as parameters of (39)

$$
\begin{aligned}
\text { domestic : } & \sigma=\sigma_{\mathrm{ATM}}, \mu=r_{d}-r_{f} \\
\text { foreign : } & \sigma=\sigma_{\mathrm{ATM}}, \mu=r_{d}-r_{f}+\sigma^{2}
\end{aligned}
$$

where $r_{d}$ and $r_{f}$ correspond to the Black-Scholes domestic and foreign interest rates. Let us denote the solution of the above PDE as $\lambda^{d}$ and $\lambda^{f}$ respectively. Finally we define $\gamma_{\mathrm{fet}}=\frac{1}{2} \frac{\left(\lambda^{d}+\lambda^{f}\right)}{\tau}$. Note that we have divided by the time to maturity in order to have a dimensionless quantity with $\gamma_{\mathrm{fet}} \in[0,1]$.

\footnotetext{
${ }^{2}$ In a window-barrier option, the barrier is activated at a time greater than the selling time of the option and deactivates before the maturity of the option.
} 

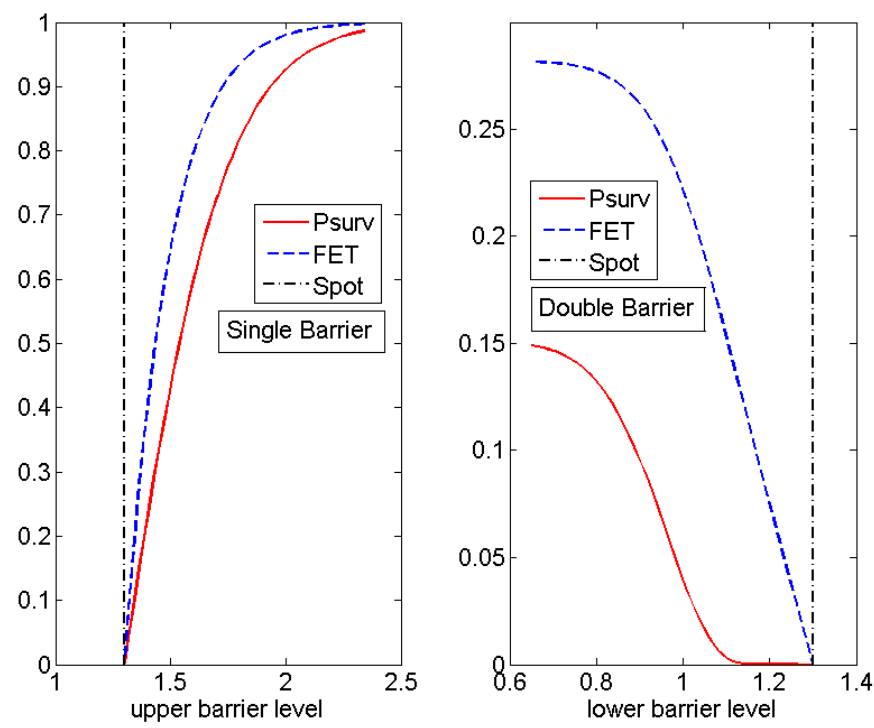

Figure 2: Comparison between $\gamma_{\text {surv }}$ and $\gamma_{\text {fet }}$ plotted against barrier level, in a single barrier case (left panel) and a double barrier case (right panel). Used Market Data: $S=1.3, \tau=1.3, r_{d}=5 \%, r_{f}=3 \%$ $\sigma_{\mathrm{ATM}}=20 \%$

\subsection{Qualitative differences between $\gamma_{\text {surv }}$ and $\gamma_{\text {fet }}$}

Although $\gamma_{\text {surv }}$ and $\gamma_{\text {fet }}$ possess similar asymptotic behavior (converging to 0 for options infinitely close to knocking-out, converging to 1 for an option infinitely far from knocking-out), they represent different quantities, and can differ substantially in intermediate situations. To support this assertion, we show in Figure 2 plots of $\gamma_{\text {surv }}$ and $\gamma_{\text {fet }}$ as a function of the barrier level, in a single-barrier and in a double-barrier case. While in the single barrier case the shapes of the two curves look similar, their discrepancy is more pronounced in the double-barrier case where the upper barrier is kept constant, and the lower barrier is progressively moved away from the spot level. For barrier levels close to the spot, there is a plateau effect in the case of $\gamma_{\text {surv }}$ which stays at zero, while $\gamma_{\text {fet }}$ seems to increase linearly. This can be explained intuitively: moving the barrier level in the close vicinity of the spot will not prevent the spot from knocking out at some point before maturity (hence $\gamma_{\text {surv }} \approx 0$ ). But although the knocking event is almost certain, the expected time at which it occurs directly depends on the barrier-spot distance.

This discussion should emphasize the importance of a careful choice between the two $\gamma$ candidates, especially when it comes to pricing double-barrier options.

There is no agreed consensus regarding which of $\gamma_{\mathrm{surv}}, \gamma_{\mathrm{fet}}$ is a better candidate for $\gamma$ in (30). Based on empirical observations, it is suggested in e.g. [23] that one uses $\gamma_{\text {surv }}$ with $a=1$ and $b=c=0.5$. Other market beliefs however favor using $\gamma_{\mathrm{fet}}$ with $a=c=1$ and $b=0$. In [26], the absence of mathematical justification for these choices is highlighted, and other adjustment possibilities are suggested, depending on the type of option at hand. In section 6 we will discuss a more systematic procedure that can allow one to calibrate the Vanna-Volga model and draw some conclusions regarding the choice of pricer. 


\subsection{Arbitrage tests}

As the Vanna-Volga method is not built on a solid bedrock but is only a practical rule-of-thumb, there is no guarantee that it will be arbitrage free. Therefore as part of the pricer one should implement a testing procedure that ensures a few basic no-arbitrage rules for barrier options (with or without strike): For example, (i) the value of a vanilla option must not be negative, (ii) the value of a single/double knock-out barrier option must not be greater than the value of the corresponding vanilla, (iii) the value of a double-knock-out barrier option must not be more expensive than either of the values of the corresponding single knock-outs, (iv) the value of a window single/double knock-out barrier option must be smaller than that of the corresponding vanilla and greater than the corresponding american single/double knock-out. For knock-in options, the corresponding no-arbitrage tests can be derived from the replication relations: (a) for single barriers, $\mathrm{KI}(\mathrm{B})=\mathrm{VAN}-\mathrm{KO}(\mathrm{B})$, where $\mathrm{B}$ represents the barrier of the option, and (b) for double-barriers, $\mathrm{KIKO}(\mathrm{KIB}, \mathrm{KOB})=\mathrm{KO}(\mathrm{KIB})-\mathrm{DKO}(\mathrm{KIB}, \mathrm{KOB})$ where KIB and KOB represent the knock-in and knock-out barrier respectively.

For touch or no-touch options, the above no-arbitrage principles are similar. One-touch options can be decomposed into a discounted cash amount and no-touch options: $\mathrm{OT}(\mathrm{B})=\mathrm{DF}-\mathrm{NT}(\mathrm{B})$ and similarly for double-one-touch options.

Based on these principles a testing procedure can be devised that amends possible arbitrage inconsistencies. We begin by using replication relations to decompose the option into its constituent parts if needed. This leaves us with vanillas and knock-out options for which we calculate the BSTV and the Vanna-Volga correction. On the resulting prices we then impose

$$
\mathrm{VAN}=\max (\mathrm{VAN}, 0) \quad \mathrm{KO}=\max (\mathrm{KO}, 0)
$$

to ensure condition (i) above. We then proceed with imposing conditions (ii)-(iv):

$$
\mathrm{KO}=\min (\mathrm{KO}, \mathrm{VAN}) \quad \mathrm{WKO}=\min (\mathrm{WKO}, \mathrm{VAN}) \quad \mathrm{WKO}=\max (\mathrm{WKO}, \mathrm{KO})
$$

while for double-knock-out options we have in addition

$$
\mathrm{DKO}=\min (\mathrm{DKO}, \mathrm{KO}(1)) \quad \mathrm{DKO}=\min (\mathrm{DKO}, \mathrm{KO}(2))
$$

where $\mathrm{KO}(1)$ and $\mathrm{KO}(2)$ represent the corresponding single knock-out options.

Note that both in the case of a double-knock-out and in that of a window-knock-out we need to create a single-knock-out instrument and launch a no-arbitrage testing on it as well.

As an example, let us consider a window knock-in knock-out option. Having an 'in' barrier this option will be decomposed to a difference between a window knock-out and a window double knockout. For the former, we will create the corresponding $\mathrm{KO}$ option while for the latter the corresponding DKO. In addition, we will also need the plain vanilla instrument. We will then price the KO and DKO separately using the Vanna-Volga pricer, ensure that the resulting value of each of these is positive (equation (45)), impose condition (iii) (equation (47D) to ensure no-arbitrage on the DKO and condition (iv) (equation (46)) to ensure that the barrier options are not more expensive than the plain vanilla.

\subsection{Sensitivity to market data}

As the FX derivatives market is rife with complex conventions it can be the case that pricing errors stemming from wrong input data have a greater impact than errors stemming from assuming wrong 
smile dynamics. This warrants discussion concerning the sensitivity of FX models with respect to market data. Already from (15) we can anticipate that the Vanna-Volga price is sensitive to the values of $\sigma_{\mathrm{RR} 25}$ and $\sigma_{\mathrm{BF} 25(2 \mathrm{vol})}$. To emphasize this dependency we will consider the following two sensitivities:

$$
\Lambda_{\mathrm{RR}}=\frac{\mathrm{d} \text { Price }}{\mathrm{d} \sigma_{\mathrm{RR} 25}} \quad \Lambda_{\mathrm{BF}}=\frac{\mathrm{d} \text { Price }}{\mathrm{d} \sigma_{\mathrm{BF} 25(2 \mathrm{vol})}}
$$

which measure the change in the Vanna-Volga price given a change in the input market data. In our tests we have used the Vanna-Volga 'survival probability' for a series of barrier levels of a OT option. Similar considerations follow by using the FET variant. The results are shown in Figure 3 where on top of the two sensitivities we superimposed the Vanna and the Volga of the option.

We notice that the two sensitivities can deviate significantly away from zero. This highlights the importance of using accurate and well-interpreted market quotes. For instance, in the 1-year USDCHF OT with the touch-level at 1.55 (BSTV price is $\approx 4 \%$ ), an error of $0.5 \%$ in the value of $\sigma_{\mathrm{BF} 25(2 \mathrm{vol})}$ would induce a price shift of $3 \%$. This is all but negligible! Thus a careful adjustment of the market data quotes is sometimes as important as the model selection.

We also see that the Volga provides an excellent estimate of the model's sensitivity to a change in the Butterfly values. Similarly, Vanna provides a good estimate of the model's sensitivity to a change in the Risk Reversal values -but only as long as the barrier level is sufficiently away from the spot. This disagreement in the region close to the spot is linked to the fact that in the Vanna-Volga recipe of section 5.1 we adjusted the Vanna contribution by the survival probability which becomes very small close to the barrier.

Figure 3 implies that for all practical purposes one should be on guard for high BS values of Vanna and/or Volga which indicate that the pricer is sensitively dependent on the accuracy of the market data.

\section{$6 \quad$ Numerical results}

In order to assess the ability of the Vanna-Volga family of models (21) to provide market prices, we compared them to a large collection of market indicative quotes. By indicative we mean that the prices we collected come from trading platforms of three major FX-option market-makers, queried without effectively proceeding to an actual trade. It is likely that the models behind these prices do not necessarily follow demand-supply dynamics and that the providers use an analytic pricing method similar to the Vanna-Volga we present here.

Our pool of market prices comprises of 3-month and 1-year options in USDCHF and USDJPY, the former currency pair typically characterized by small $\sigma_{\mathrm{RR}}$ values, while the latter by large ones. In this way we expect to span a broad range of market conditions. For each of the four maturity/currency pair combinations we select four instrument types, representative of the first generation exotics family: Reverse-Knock-Out call (RKO), One-Touch (OT), Double-Knock-Out call (DKO), and Double-OneTouch (DOT). In the case of single barrier options (RKO and OT), 8 barrier levels are adjusted, mapping to probabilities of touching the barrier that range from $10 \%$ to $90 \%$. In the case of the RKO call, the strike is set At-The-Money-Spot. In the case of two-barrier options (DKO and DOT), since it is practically impossible to fully span the space of the two barriers we selected the following subspace: (i) we fix the lower barrier level in such a way that it has a $10 \%$ chance of being hit, then select 5 upper barrier levels such that the overall hitting probabilities (of any of the 2 barriers) range approximately from $15 \%$ to $85 \%$. (ii) We repeat the same procedure with a fixed upper barrier level, and 5 adjusted lower barrier levels. 

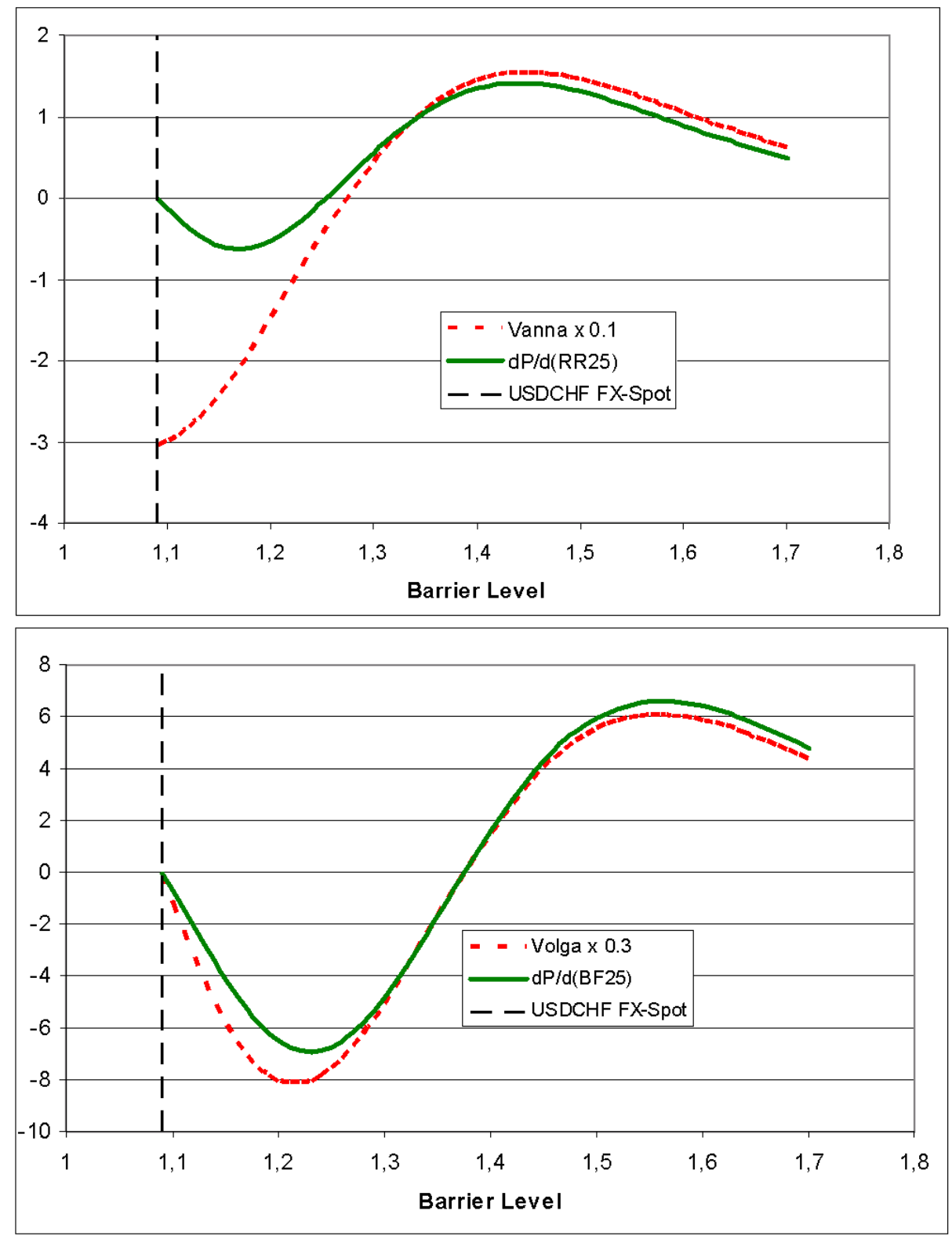

Figure 3: Sensitivity of the Vanna-Volga price with respect to input market data for a OT option. Top: Comparison between the Vanna (BSTV) and $\Lambda_{\mathrm{RR}}$. Bottom: Comparison between the Volga (BSTV) and $\Lambda_{\mathrm{BF}}$. We see that the two Greeks provide a good approximation of the two model sensitivities. 
In summary, our set of data consists of the cross product $\mathcal{F}$ of the sets

$$
\begin{aligned}
\text { currency pair : } & A=\{\mathrm{USDJPY}, \mathrm{USDCHF}\} \\
\text { maturity period : } & B=\{3 m, 1 y\} \\
\text { option type : } & C=\{\mathrm{RKO}, \mathrm{OT}, \mathrm{DKO}, \mathrm{DOT}\} \\
\text { barrier value : } & D=\left\{B_{1}, \ldots, B_{n}\right\}
\end{aligned}
$$

where $n=10$ for double-barrier options and $n=8$ for single barrier ones.

In order to maintain coherence, each of the two data sets were collected in a half-day period (in Nov. 2008 for USDJPY, in Jan. 2009 for USDCHF).

Thus in total our experiments are run over the set of models

$$
\text { models : } \quad E=\left\{\mathrm{VV}_{\text {surv }}, \mathrm{VV}_{\text {fet }}\right\}
$$

\subsection{Definition of the model error}

In order to focus on the smile-related part of the price of an exotic option, let us define for each instrument $i \in \mathcal{F}$ from our pool of data (49) the 'Model Smile Value' (MODSV) and the 'Market Smile Value' (MKTSV) as the difference between the price and its Black-Scholes Theoretical Value (BSTV):

$$
\begin{aligned}
& \operatorname{MODSV}_{i}^{k}=\text { Model Price }{ }_{i}^{k}-\mathrm{BSTV}_{i}^{k} \quad k=1, \ldots, N_{\text {mod }} \\
& \operatorname{MKTSV}_{i}^{k}=\text { Market Price }_{i}^{k}-\mathrm{BSTV}_{i}^{k} \quad k=1, \ldots, N_{\mathrm{mkt}}
\end{aligned}
$$

(where market prices are taken as the average between bid and ask prices) and where $N_{\text {mod }}=4$ is the number of models we are using and $N_{\mathrm{mkt}}=3$ the number of FX market makers where the data is collected from. Let us also define the average, minimum and maximum of the market smile value:

$$
\begin{aligned}
& \overline{\operatorname{MKTSV}_{i}}=\frac{1}{N_{\mathrm{mkt}}} \sum_{k \leq N_{\mathrm{mkt}}} \operatorname{MKTSV}_{i}^{k} \\
& \min _{i}=\min _{k \in N_{\mathrm{mkt}}} \operatorname{MKTSV}_{i}^{k}, \quad \max _{i}=\max _{k \in N_{\mathrm{mkt}}} \operatorname{MKTSV}_{i}^{k}
\end{aligned}
$$

We now introduce an error measure quantifying the ability of a model to describe market prices. This function is defined as a quadratic sum over the pricing error :

$$
\varepsilon_{k}=\sum_{i \in \mathcal{F}}\left(\frac{\operatorname{MODSV}_{i}^{k}-\overline{\operatorname{MKTSV}_{i}}}{\max _{i}-\min _{i}}\right)^{2}
$$

The error is weighted by the inverse of the market spread, defined as the difference between the maximum and the minimum mid market price for a given instrument. This setup is designed (i) to yield a dimensionless error measure that can be compared across currency pairs and the type of options, (ii) to link the error penalty to the market coherence: a pricing error on an instrument which is priced very similarly by the 3 market providers will be penalized more heavily than the same pricing error where market participants exhibit large pricing differences among themselves. Note also that the error is defined as the deviation from the average market price. 


\subsection{Shortcomings of common stochastic models in pricing exotic options}

Before trying to calibrate the Vanna-Volga weighting factors $p_{\text {vanna }}$ and $p_{\text {volga }}$, we investigate how the Dupire local vol [6] and the Heston stochastic vol [7] models perform in pricing our set of selected exotic instruments (for a discussion on the pricing of barrier instruments under various model frameworks, see for example [13, 14, 15]). In order to obtain a fast and reliable calibration for Heston, the price of call options is numerically computed through the characteristic function [1, 11, and Fourier inversion methods. To price exotic options, Heston dynamics is simulated by Monte Carlo, using a QuadraticExponential discretization scheme [2].

Figure 4 shows the MODSV of a 1-year OT options in USDCHF (lower panel) and USDJPY (upper panel), as the barrier moves away from the spot level $\left(S_{t}=95.47\right.$ for USDJPY and $S_{t}=1.0902$ for USDCHF). At first inspection, none of the models gives satisfactory results.

\begin{tabular}{|c|c|c|c|}
\hline & & USDCHF & USDJPY \\
\hline \multirow{7}{*}{ 1-Year } & $\mathrm{RKO}$ & Heston & Dupire \\
\hline & OT & Heston & Heston \\
\hline & $\mathrm{DKO}(\mathrm{Up})$ & Dupire & Dupire \\
\hline & DKO(Down) & Heston & Heston \\
\hline & DOT(Up) & Heston & Dupire \\
\hline & DOT(Down) & Heston & Dupire \\
\hline & global & Heston $(\varepsilon=62)$ & Dupire $(\varepsilon=96)$ \\
\hline \multirow{7}{*}{ 3-Month } & RKO & Heston & Dupire \\
\hline & OT & Heston & Dupire \\
\hline & $\overline{\mathrm{DKO}(\mathrm{Up})}$ & Heston & Dupire \\
\hline & DKO(Down) & Heston & Heston \\
\hline & $\mathrm{DOT}(\mathrm{Up})$ & Heston & Dupire \\
\hline & DOT(Down) & Heston & Heston \\
\hline & global & Heston $(\varepsilon=65)$ & Dupire $(\varepsilon=73)$ \\
\hline
\end{tabular}

Table 2: Heston stochastic vol Vs. Dupire local vol in pricing 1st generation exotics.

Using the error measure defined above, we now try to formalize the impressions given by our rough inspection of Figure 4. For each combination of the instruments in 49 we determine which of Dupire local vol or Heston stochastic vol gives better market prices. The outcome of this comparison is given in the Table 2 .

This table suggests that -in a simplified world where exotic option prices derive either from Dupire local vol or from heston stochastic vol dynamics- an FX market characterized by a mild skew (USDCHF) exhibits mainly a stochastic volatility behavior, and that FX markets characterized by a dominantly skewed implied volatility (USDJPY) exhibit a stronger local volatility component. This confirms that calibrating a stochastic model to the vanilla market is by no mean a guarantee that exotic options will be priced correctly [21], as the vanilla market carries no information about the smile dynamics.

In reality the market dynamics could be better approximated by a hybrid volatility model that contains both some stochastic vol dynamics and some local vol one. This model will be quite rich but the calibration can be expected to be considerably hard, given that it tries to mix two very different smile dynamics, namely an 'absolute' local-vol one with a 'relative' stochastic vol one. For a discussion 

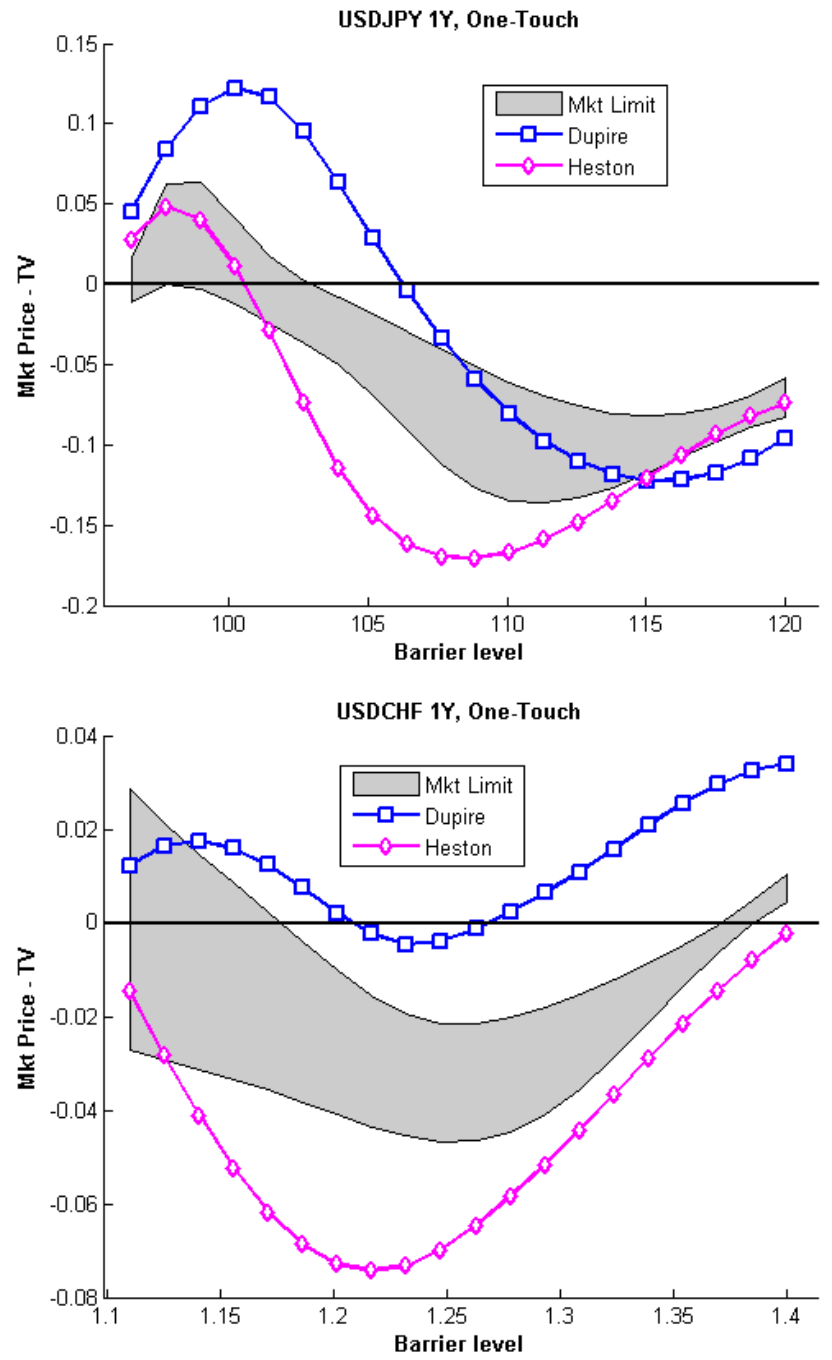

Figure 4: Smile value vs. barrier level; comparison of the various models for OT 1-year options in USDCHF (bottom) and USDJPY (top). Market limits are indicated with black solid lines. 
of such a model, we refer the reader to [14].

At this stage one has the option to either go for the complex hybrid model or for the more heuristic alternative method like the Vanna-Volga. In this paper we present the latter.

\subsection{Vanna-Volga calibration}

The purpose of this section is to provide a more systematic approach in selecting the coefficients $a, b$ and $c$ in $(30)$ and thus the factors $p_{\text {vanna }}$ and $p_{\text {volga }}$.

We first determine the optimal values of coefficients $a, b$ and $c$ in the sense of the least error (52), where the sum extends to all instruments and to the two maturities (e.g. a single error function per currency pair). This problem can readily be solved using standard linear regression tools, as $a, b$ and $c$ appear linearly in the VV correction term, but most standard solver algorithms would as well do the job. This optimization problem is solved four times in total, for USDCHF with $\gamma_{\text {surv }}$ and $\gamma_{\text {fet }}$, and for USDJPY with $\gamma_{\text {surv }}$ and $\gamma_{\text {fet }}$. Let us point out that such a calibration is of course out of the question in a real trading environment: collecting such an amount of market data each time a recalibration is deemed necessary would be way too time-consuming. Our purpose is simply to determine some limiting cases, to be used as benchmarks for the results of a more practical calibration procedure discussed later. Table 3 presents these optimal solutions, indicating the minimum error value, along with the value of the optimal coefficients $a, b$ and $c$.

\begin{tabular}{|c|c|c|}
\cline { 2 - 3 } \multicolumn{1}{c|}{} & USDCHF & USDJPY \\
\hline$\gamma_{\text {surv }}$ & $\varepsilon=\mathbf{1 9 . 7}$ & $\varepsilon=\mathbf{1 5 . 6}$ \\
& $a=0.54, b=0.29, c=0.14$ & $a=0.74, b=0.7, c=0.05$ \\
\hline$\gamma_{\text {fet }}$ & $\varepsilon=\mathbf{1 8 . 2}$ & $\varepsilon=\mathbf{1 4 . 7}$ \\
& $a=0.49, b=0.35, c=0.01$ & $a=0.54, b=0.17, c=0.52$ \\
\hline
\end{tabular}

Table 3: Overall pricing error, calibration on entire market price set.

Comparing the above error numbers to those of Table 2, it seems possible that the Vanna-Volga models have the potential to outperform the Dupire or Heston models.

We now discuss a more practical calibration approach, where the minimization is performed on OT prices only. The question we try to answer is: 'Can we calibrate a VV model on OT market prices, and use this model to price other first generation exotic products ?'. Performing this calibration with 3 parameters to optimize will certainly improve the fitting of OT prices, but at the expense of destroying the fitting quality for the other instruments (in the same way that performing a high-order linear regression on a set of data points, will produce a perfect match on the data points and large oscillations elsewhere). This is confirmed by the results of Table 4 , showing how the error (on the entire instrument set) increases with respect to the error of Table 3 when the optimization is performed on the OT subset only.

\begin{tabular}{|c|c|c|}
\cline { 2 - 3 } \multicolumn{1}{c|}{} & USDCHF & USDJPY \\
\hline$\gamma_{\text {surv }}$ & $\varepsilon=\mathbf{4 4 . 6}$ & $\varepsilon=\mathbf{2 6 . 8}$ \\
\hline$\gamma_{\mathrm{fet}}$ & $\varepsilon=\mathbf{4 7 . 2}$ & $\varepsilon=\mathbf{8 5}$ \\
\hline
\end{tabular}

Table 4: Overall pricing error, calibration on OT prices only. 
For robustness reasons, it is thus desirable to reduce the space of free parameters in the optimization process. We consider the following two constrained optimization setups: (i) $a=c, b=0$ and (ii) $b=c=0.5 \cdot a$, which are re-scaled versions of the market practices described in section 5.3 . Needless to say that the number of possible configurations here are limited only by one's imagination. Our choice is dictated mainly by simplicity, namely we have chosen to keep a single degree of freedom. The results are presented in Table 5 where we compare four possible configurations measured over all instruments and maturity periods for our two currency pairs.

\begin{tabular}{|l|c|c|c|c|}
\cline { 3 - 5 } \multicolumn{2}{c|}{} & USDCHF & USDJPY & Total error \\
\hline configuration 1 & $\gamma_{\text {surv }}$ & $\varepsilon=21.8$ & $\varepsilon=28.4$ & $\varepsilon=50.2$ \\
& $b=c=0.5 \cdot a$ & $a=0.43$ & $a=0.72$ & \\
\hline configuration 2 & $\gamma_{\mathrm{fet}}$ & $\varepsilon=21.2$ & $\varepsilon=26$ & $\varepsilon=47.2$ \\
& $b=c=0.5 \cdot a$ & $a=0.39$ & $a=0.63$ & \\
\hline configuration 3 & $\gamma_{\mathrm{surv}}$ & $\varepsilon=32.2$ & $\varepsilon=72.1$ & $\varepsilon=104.3$ \\
& $a=c, b=0$ & $a=0.51$ & $a=0.69$ & \\
\hline configuration 4 & $\gamma_{\mathrm{fet}}$ & $\varepsilon=24.3$ & $\varepsilon=19.4$ & $\varepsilon=43.7$ \\
& $a=c, b=0$ & $a=0.42$ & $a=0.60$ & \\
\hline
\end{tabular}

Table 5: Overall pricing error, constrained calibration on OT prices only.

As there is no sound mathematical (or economical) argument to prefer one configuration over another, we therefore choose the least-error configuration, namely configuration $n^{\circ} 4$. One additional argument in favor of $\gamma_{\mathrm{fet}}$ is that it accommodates window-barrier options without further adjustment. This is not the case of $\gamma_{\text {surv }}$ where some re-scaling should be used to account for the start date of the barrier (when the barrier start date is very close to the option maturity, the path-dependent character vanishes and the full $\mathrm{VV}$ correction applies i.e. $p_{\text {vanna }}=p_{\text {volga }}=1$ even for small $\gamma_{\text {surv values). }}$.

In Figure 5 we show results from the calibration of the Vanna-Volga method. It is based on minimizing the error (52) of (i) all instruments of the data pool and while having all coefficients $a, b, c$ of $\gamma_{\mathrm{fet}}$ free and (ii) of one-touch options only and with configuration $n^{o} 4$ (thus, we have chosen $\gamma_{\mathrm{fet}}$ with $a=c, b=0$ ). We see that in general calibration (i) performs better in the sense that it falls well within the shaded area that corresponds to the limits of the market price as provided by the FX market makers. This is not surprising as this calibration is meant to be the most general and flexible. However this is clearly an impractical calibration procedure. On the contrary, the calibration method (ii) that is based on quotes from a single exotic instrument has practical advantages and appears in good agreement with that of (i). Finally note that these pictures are representative of our results in general.

\section{Conclusion}

The Vanna-Volga method is a popular pricing tool for FX exotic options. It is appealing to both traders, due to its clear interpretation as a hedging tool, and to quantitative analysts, due to its simplicity, ease of implementation and computational efficiency. In its simplest form, the Vanna-Volga recipe assumes that smile effects can be incorporated to the price of an exotic option by inspecting the effect of the smile on vanilla options. Although this recipe, outlined in (15), turns out to give often uncomfortably large values, there certainly is a silver lining there. This has led market practitioners 

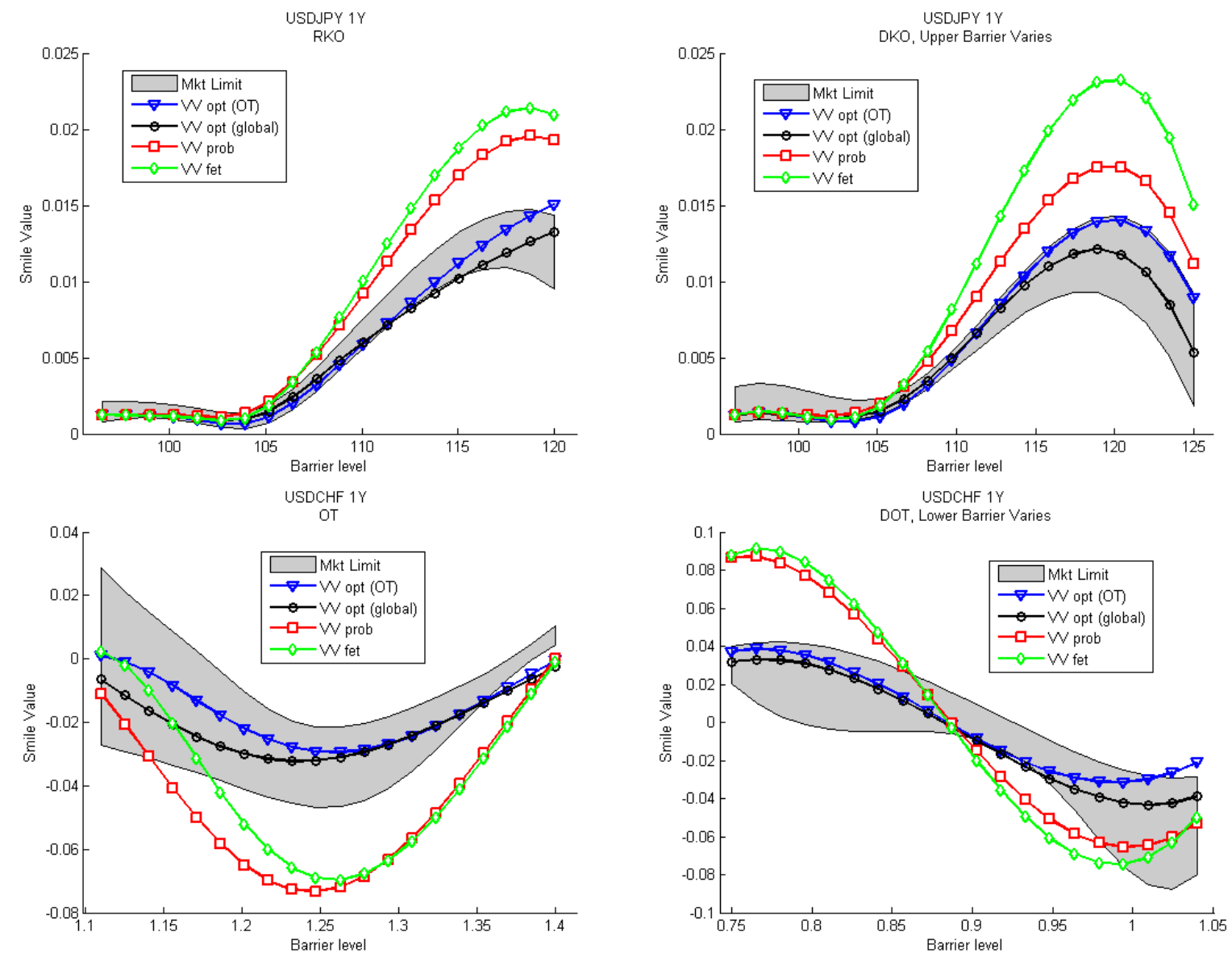

Figure 5: Results from calibrating the Vanna-Volga method on (i) all instruments of our data pool (marked as 'VV opt (global))', (ii) one-touch options only (marked as 'VV opt (OT))'. The results of the two calibrations do not differ significantly while the latter is naturally more convenient from a practical perspective. The shaded areas correspond to the region within which market makers provide their indicative mid price. For comparison we also show the non-calibrated Vanna-Volga methods based on the 'survival probability' and the 'first exit time'. 
to consider several ways to adapt the Vanna-Volga method. In this article we have reviewed some commonly used adaptations based on rescaling the Vanna-Volga correction by a function of either the 'survival probability' or the 'first exit time'. These variations provide prices that are more in line with the indicative ones given by market makers.

We have attempted to improve the Vanna-Volga method further by adjusting the various rescaling factors that are involved. This optimization is based on simple data analysis of one-touch options that are obtained from renowned FX platforms. It involves a single optimization variable and as a result we find that for a wide range of exotic options, maturity periods and currency pairs it leads to prices that agree well with the market mid-price.

The FX derivatives community, perhaps more than any other asset class, lives on a complex structure of quote conventions. Naturally, a wrong interpretation of the input market data cannot lead to the correct results. To this end, we have presented some relevant FX conventions regarding smile quotes and we have tested the robustness of the Vanna-Volga method against the input data. It appears that the values of Vanna and Volga provide a good indication of the VV price sensitivity to a change in smile input parameters.

\section{References}

[1] H. Albrecher, P. Mayer, W. Schoutens and J. Tistaert, 'The little Heston trap', Wilmott Magazine (January 2007) 83-92.

[2] L. Andersen, 'Efficient Simulation of the Heston Stochastic Volatility Model', Available at SSRN: http://ssrn.com/abstract=946405 (January 23, 2007).

[3] F. Black and M. Scholes, 'The pricing of Options and Corporate Liabilities', Journal of Political Economy 81 (1973) 637-654.

[4] D. Brigo and F. Mercurio, 'Interest Rate Models - Theory and Practice With Smile, Inflation and Credit', Springer (2007).

[5] A. Castagna and F. Mercurio, 'The Vanna-Volga method for implied volatilities', Risk (January 2007) 106-111.

[6] B. Dupire, 'Pricing with a smile', Risk (January 1994) 18-20.

[7] S. L. Heston, 'A closed-form solution for options with stochastic volatility with applications to bond and currency options', Rev Fin Studies 6 (1993) 327-343.

[8] J. C. Hull, 'Options, Futures and Other Derivatives', Prentice Hall Series in Finance (2006) 6th edition.

[9] E. Reiner and M. Rubinstein, 'Unscrambling the Binary Code', Risk October 1991, 75-83.

[10] E. Reiner and M. Rubinstein, 'Breaking down the barriers', Risk September 1991, 28-35.

[11] P. Jäckel and C. Kahl, 'Not-So-Complex Logarithms in the Heston Model', Wilmott Magazine (September 2005) 94-103.

[12] N. Kunitomo and M. Ikeda, 'Pricing Options with Curved Boundaries', Mathematical Finance 4 (1992) 275-298. 
[13] A. Lipton, 'Mathematical Methods for Foreign Exchange: A Financial Engineer's Approach', World Scientific (2001).

[14] A. Lipton, 'The vol smile problem', Risk Magazine (2002) 15, n. 2, 61-65.

[15] A. Lipton and W. McGhee, 'Universal barriers', Risk Magazine (2002) 15, n. 5, 81-85.

[16] T. Fisher, 'Variations on the Vanna-Volga adjustment', Bloomberg Quantitative Research and Development FX Team' (2007), private communication.

[17] Y. Shkolnikov, 'Generalized Vanna-Volga Method and its Applications', Available at SSRN: http://ssrn.com/abstract=1186383 (June 25, 2009).

[18] R. C. Merton, 'Theory of Rational Option Pricing', Bell Journal of Economics and Management Science 4 (1973) 141-183.

[19] E. Derman, 'Regimes of Volatility', Quantitative Strategies Research Notes, Goldman Sachs, (1999).

[20] M. Rubinstein and E. Reiner, 'Exotic Options', Working paper, UC Berkeley (1992).

[21] W. Schoutens, E. Simons, and J. Tistaert, 'A Perfect calibration! Now what?', Wilmott Magazine (March 2004).

[22] S. E. Shreve, 'Stochastic Calculus for Finance II: Continuous-Time Models', Springer-Finance (2004).

[23] H. J. Stein, 'FX Market Behavior and Valuation', Available at SSRN: http://ssrn.com/abstract=955831 (December 13, 2006).

[24] P. Wilmott, 'Paul Wilmott on Quantitative Finance', John Wiley \& Sons (2006).

[25] U. Wystup, 'FX Options and structured products', Wiley Finance (2006).

[26] U. Wystup, 'Vanna-Volga Pricing', MathFinance AG (June 2008) Available at http://www.mathfinance.com/wystup/papers/wystup_vannavolga_eqf.pdf.

[27] U. Wystup, 'The market price of one-touch options in foreign exchange markets', Derivatives Week (2003) 12(13), 1-4. 


\section{A Definitions of notation used}

\begin{tabular}{||l||l||}
\hline$t$ & date of today \\
$\tau$ & maturity date \\
$S_{t}$ & time to expiry (expressed in years) \\
$K$ & spot today \\
$r_{f / d}(t)$ & strike \\
$\sigma$ & foreign/domestic interest rates \\
$\mathrm{DF}_{f / d}(t, T)=\exp \left[-r_{f} / d\right]$ & volatility of the FX-spot \\
$F=S_{t} \mathrm{DF} f(t, T) / \mathrm{DF}_{d}(t, T)$ & foreign/domestic discount factor \\
$d_{1}=\frac{\ln \frac{F}{K}+\frac{1}{2} \sigma^{2} \tau}{\sigma \sqrt{\tau}}$ & forward price \\
$d_{2}=\frac{\ln \frac{F}{K}-\frac{1}{2} \sigma^{2} \tau}{\sigma \sqrt{\tau}}$ & \\
$N(z)=\int_{-\infty}^{z} d x \frac{1}{\sqrt{2 \pi}} e^{-\frac{1}{2} x^{2}}$ & \\
\hline
\end{tabular}

Table 6: List of abbreviations.

\section{B Premium-included Delta}

For correctly calculating the Delta of an option it is important to identify which of the currencies represents the risky asset and which one represents the riskless payment currency.

Let us consider a generic spot quotation in terms Ccy1-Ccy2 representing the amount of Ccy2 per unit of Ccy1. If the (conventional) premium currency is Ccy2 (e.g. USD in EURUSD) then by convention the 'risky' asset is Ccy1 (EUR in this case) while Ccy2 refers to the risk-free one. In this case the standard Black-Scholes theory applies and the Delta expressed in Ccy1 is found by a simple differentiation of (1): $\Delta_{\mathrm{BS}}=\mathrm{DF}_{f}(t, T) N\left(d_{1}\right)$. This represents an amount of Ccy1 to sell if one is long a Call.

If, however, the premium currency is Ccy1 (e.g. USD in USDJPY) then Ccy2 is considered as the risky asset while Ccy1 the risk-free one. In this case, the value of the Delta is $\Delta=S_{t} \Delta_{\mathrm{BS}}-\mathrm{Call}_{t}$, where Call $t$ is the premium in units of Ccy2 while $\Delta$ and $\Delta_{\mathrm{BS}}$ are expressed in their 'natural' currencies; Ccy2 and Ccy1, respectively (for lightening notations, we omit the time index $t$ in $\Delta$ and $\Delta_{\mathrm{BS}}$ ). In this case $\Delta$ represents an amount of Ccy2 to buy. This relation can be seen by the following argument. First note that the Black-Scholes vanilla price of a call option is

$$
\mathrm{Call}_{t}=\mathrm{DF}_{d}(t, T) \mathbb{E}^{d}\left[\max \left(S_{T}-K, 0\right)\right]
$$

where the index ' $d$ ' implies that we are referring to the domestic risk-neutral measure, i.e. we take the domestic money-market (MM) unit $1 / \mathrm{DF}_{d}(t, T)$ as numeraire. If we now wish to express (53) into a measure where the numeraire is the foreign money-market account then

$$
\mathrm{Call}_{t}=\mathrm{DF}_{d}(t, T) \mathbb{E}^{d}\left[\max \left(S_{T}-K, 0\right)\right]=\mathrm{DF}_{d}(t, T) \mathbb{E}^{f}\left[\frac{d Q^{d}}{d Q^{f}}(T) \max \left(S_{T}-K, 0\right)\right]
$$


where we introduced the Radon-Nikodym derivative (see for example [4, 22])

$$
\frac{d Q^{d}}{d Q^{f}}(T)=\frac{\mathrm{DF}_{f}(t, T)}{\mathrm{DF}_{d}(t, T)} \frac{S_{t}}{S_{T}}
$$

This equality allows us to derive the foreign-domestic parity relation

$$
\mathrm{Call}_{t}=\mathrm{DF}_{d}(t, T) \mathbb{E}^{d}\left[\max \left(S_{T}-K, 0\right)\right]=\mathrm{DF}_{f}(t, T) S_{t} K \mathbb{E}^{f}\left[\max \left(\frac{1}{K}-\frac{1}{S_{T}}, 0\right)\right]
$$

where both sides are expressed in units of Ccy2 (for a unit nominal amount in Ccy1). The above foreign/domestic relation illustrates the fact that in FX any derivative contract can be regarded either from a domestic or from a foreign standpoint. However the contract value is unique. On the contrary, the Delta of the option depends on the adopted perspective. In 'domestic' vs. 'foreign' worlds we have respectively

$$
\Delta_{\mathrm{BS}}=\frac{\partial \mathrm{Call}_{t}}{\partial S_{t}} \quad \Delta=-\frac{\partial \frac{\mathrm{Call}_{t}}{S_{t}}}{\partial \frac{1}{S_{t}}}
$$

where the first equation is expressed in units of Ccy1 (to sell) while the second in units of Ccy2 (to buy). Setting up a Delta hedged portfolio (at time $t$ ) in the 'foreign' world implies that at any instant of time $t^{\prime}>t$, where $t$ represents today, the portfolio in Ccy1

$$
\Pi_{t^{\prime}}=\frac{\text { Call }_{t^{\prime}}}{S_{t^{\prime}}}+\frac{\Delta}{S_{t^{\prime}}}
$$

will be insensitive to variations of the spot $S_{t}$. From $\partial \Pi_{t^{\prime}} /\left.\partial S_{t^{\prime}}\right|_{t^{\prime}=t}=0$ we then find

$$
\Delta=S_{t} \Delta_{\mathrm{BS}}-\mathrm{Call}_{t}
$$

\begin{tabular}{|c|c|c|}
\hline & USD world & JPY world \\
\hline Local MM unit & 1 USD & 1 JPY \\
\hline Risky asset & JPY & USD \\
\hline Contract value in local MM units & $\mathrm{Call}_{t} / S_{t}$ & Call $_{t}$ \\
\hline Risky asset in local MM units & $1 / S_{t}$ & $S_{t}$ \\
\hline$\Delta$ hedge: amount of risky asset to short & $\frac{\partial \frac{\mathrm{Call}_{t}}{S_{t}}}{\partial \frac{1}{S_{t}}}=\mathrm{Call}_{t}-S_{t} \Delta_{\mathrm{BS}}(\mathrm{JPY})$ & $\frac{\partial \text { Call }}{\partial S_{t}}=\Delta_{\mathrm{BS}}(\mathrm{USD})$ \\
\hline Amount of USD to short & $\tilde{\Delta}=-\frac{\partial \frac{\text { Call }}{S_{t}}}{\partial \frac{1}{S_{t}}} \frac{1}{S_{t}}=\Delta_{\mathrm{BS}}-\frac{1}{S_{t}}$ Call (USD) & $\frac{\partial \mathrm{Call}}{\partial S_{t}}=\Delta_{\mathrm{BS}}(\mathrm{USD})$ \\
\hline
\end{tabular}

Note that FX convention dictates that the $\Delta$ is always quoted in units of Ccy1 (regardless of the currency to which the premium is paid), hence to obtain the relation mentioned in section 3.1 we simply take $\Delta \rightarrow \tilde{\Delta}=\Delta / S_{t}$. Table 7 provides a vis-à-vis of the various quantities under the two perspectives for an option in USDJPY with the Spot $S_{t}$ defined as the amount of JPY per USD.

Table 7: Delta hedge calculation, domestic versus foreign world. 